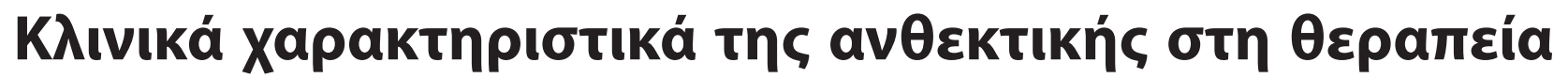

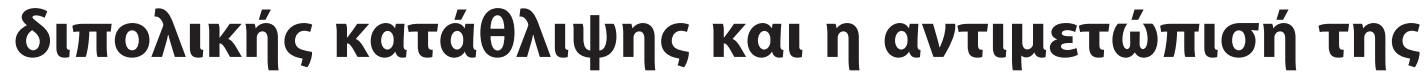

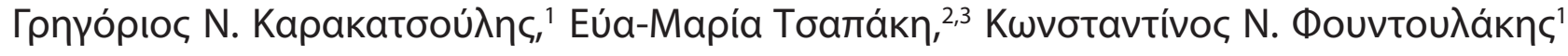

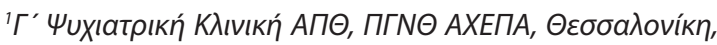

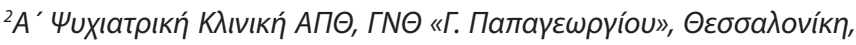

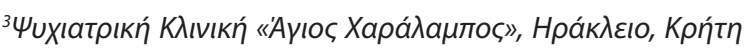

\begin{abstract}
ПЕРІАНЧН

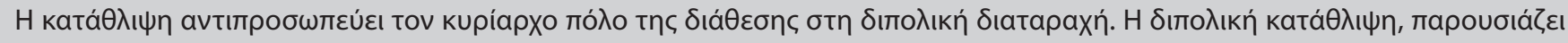

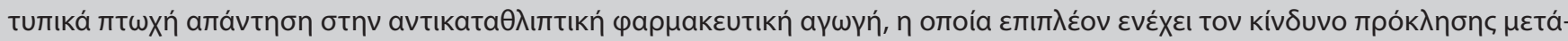

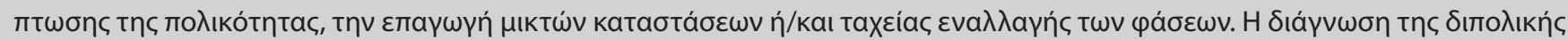

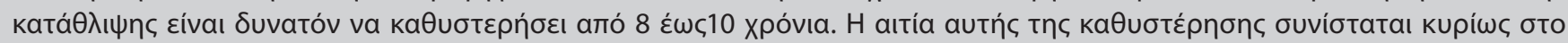

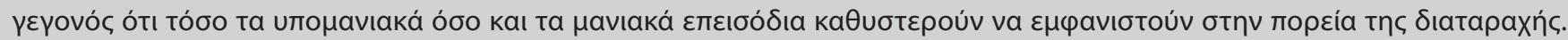

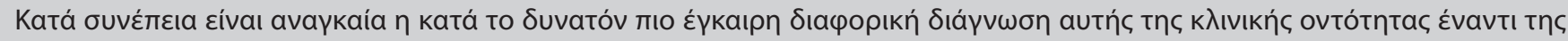

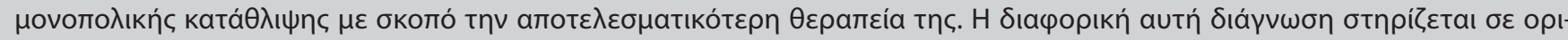

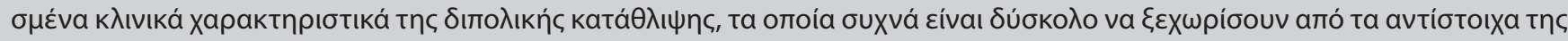

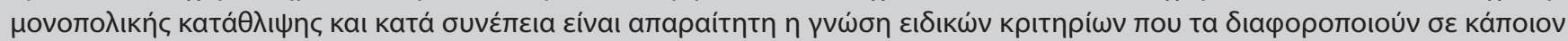

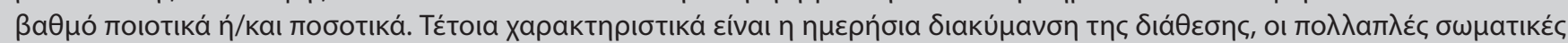

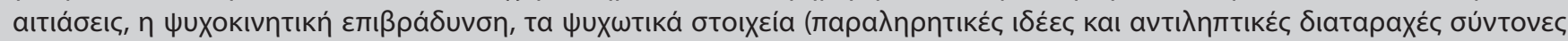

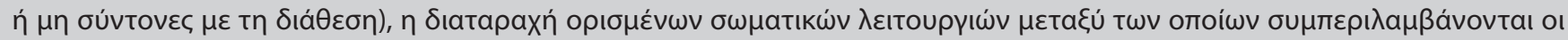

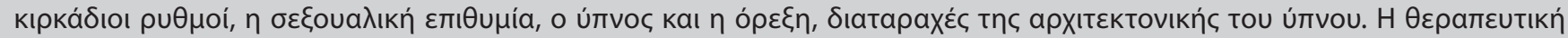

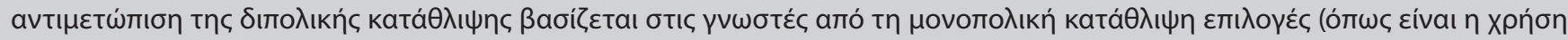

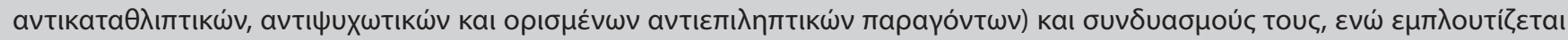

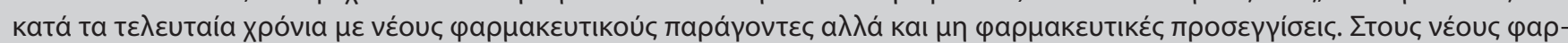

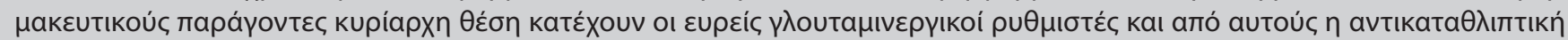

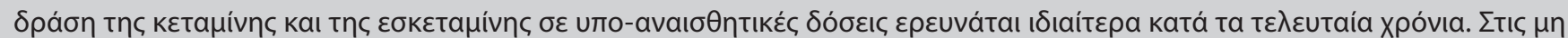

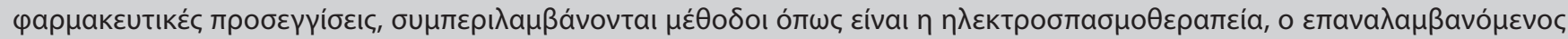

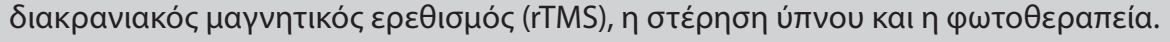

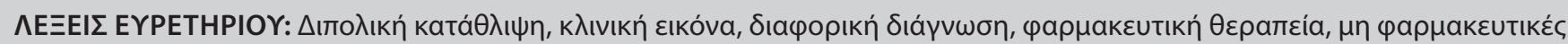

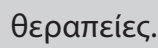

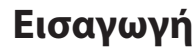

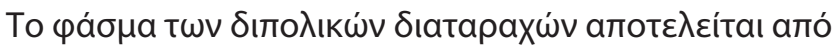

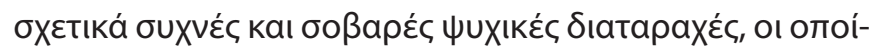

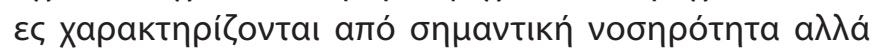

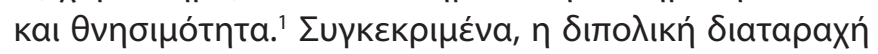

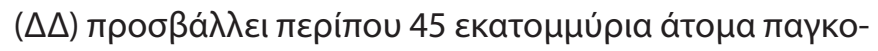

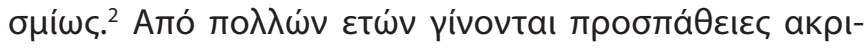

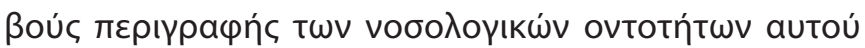

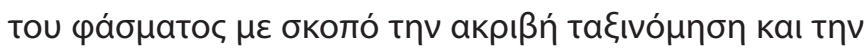

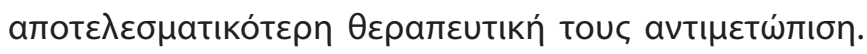

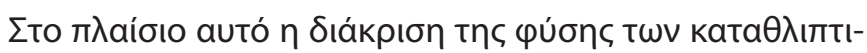

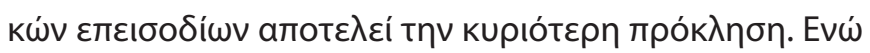




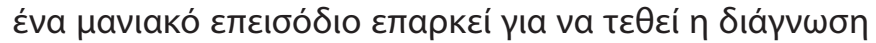

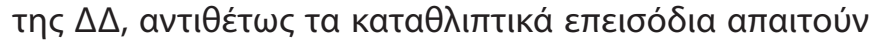

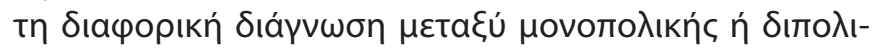
кńৎ кatá $\theta \lambda ı \psi \eta \varsigma(\Delta K){ }^{3}$

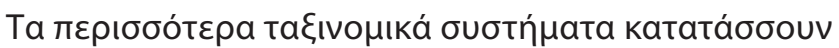

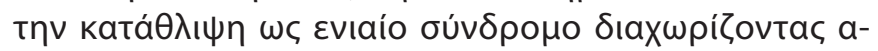

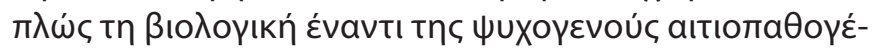

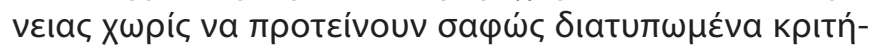

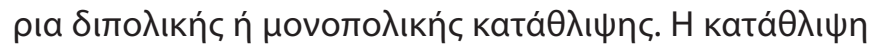

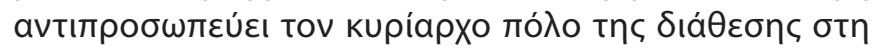

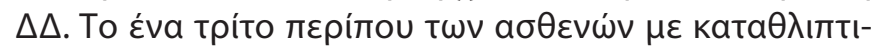

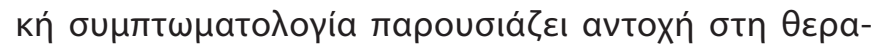

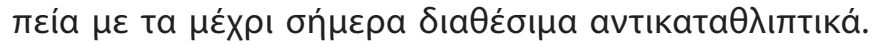

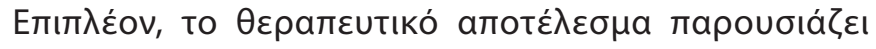

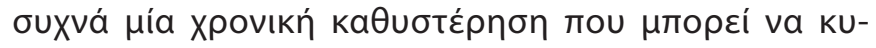

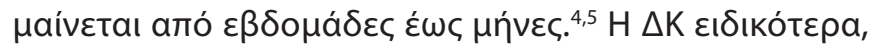

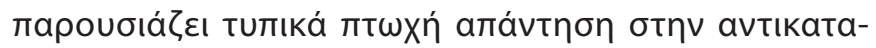

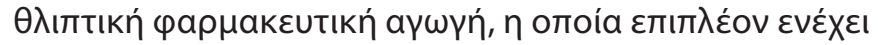

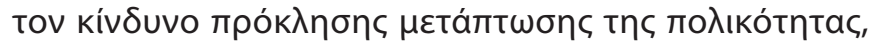

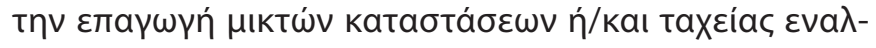

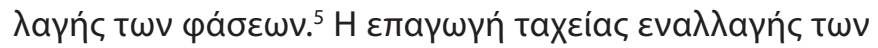

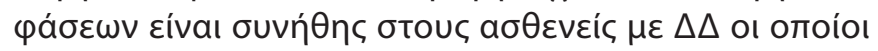

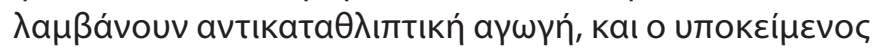

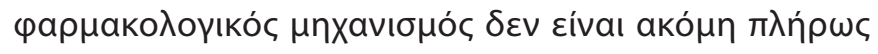
katavontóç. ${ }^{6}$

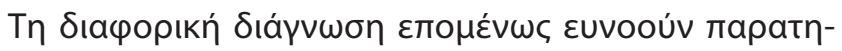

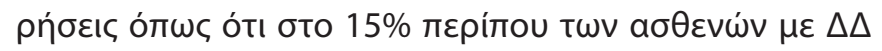

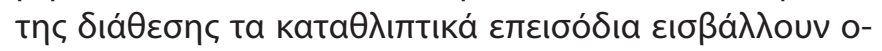

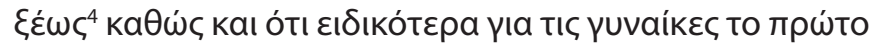

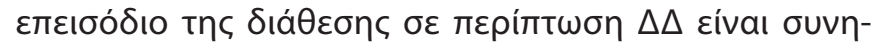

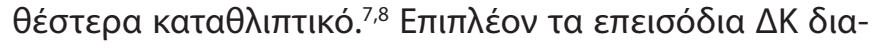

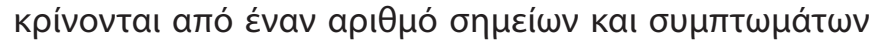

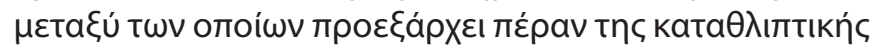

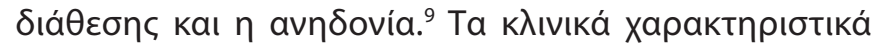

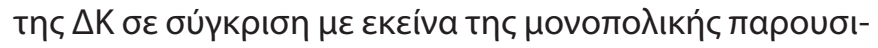

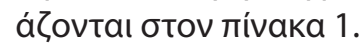

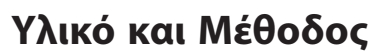

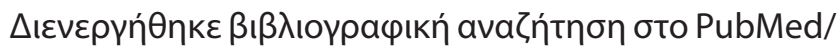

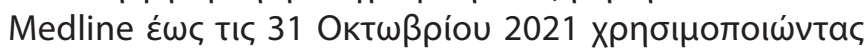

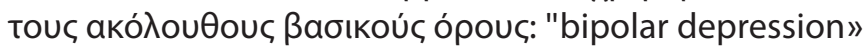
['Opoı MeSH] AND "clinical picture" ['Opoı MeSH] AND "treatment resistant" ['O $\alpha a$ тa $\pi \varepsilon \delta i ́ a]$ AND "differential

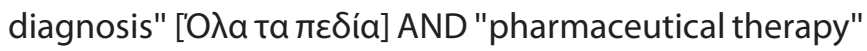

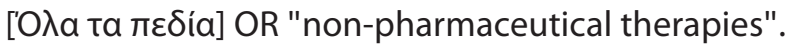

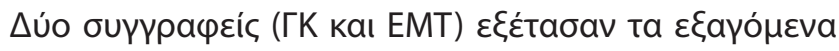

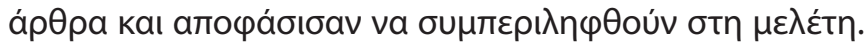

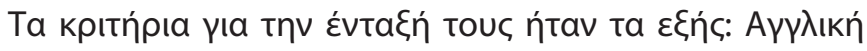

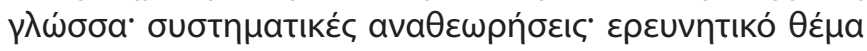

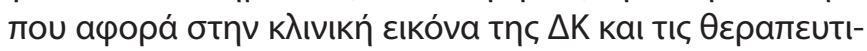

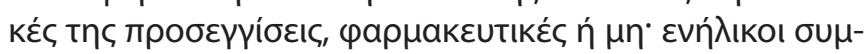

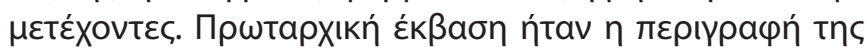

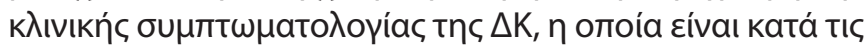

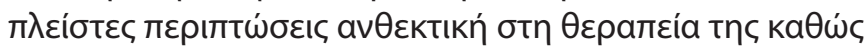

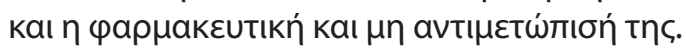

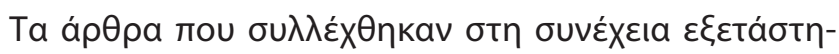

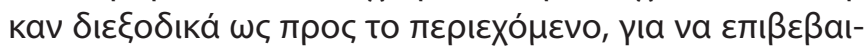

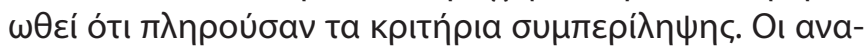

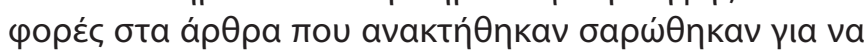

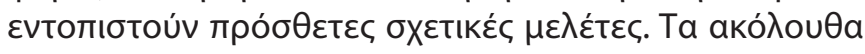

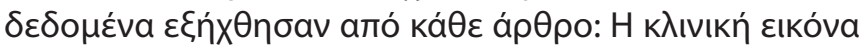

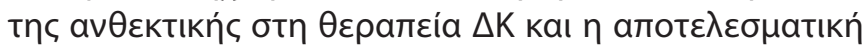

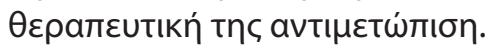

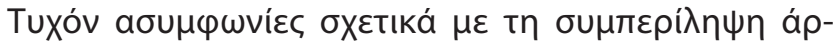

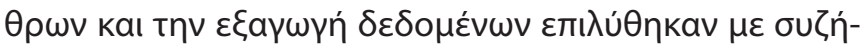

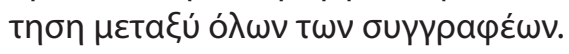

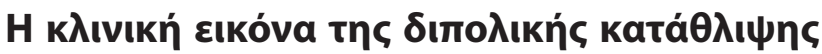

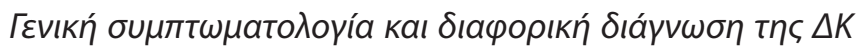

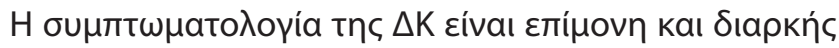

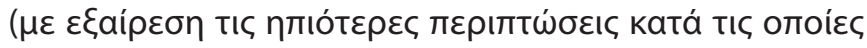

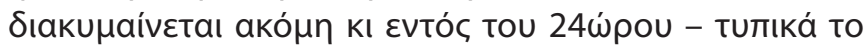

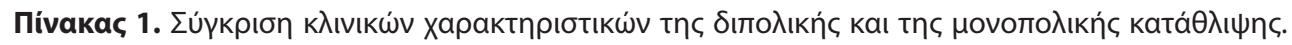

\begin{tabular}{|c|c|}
\hline 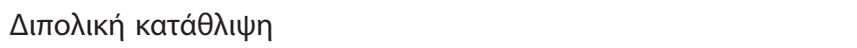 & 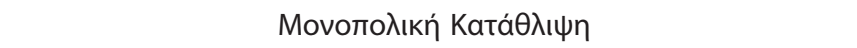 \\
\hline 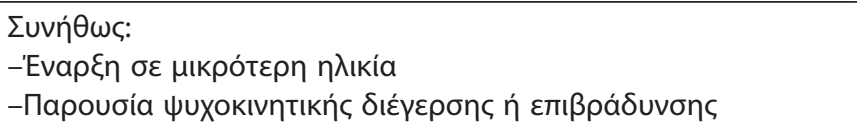 & 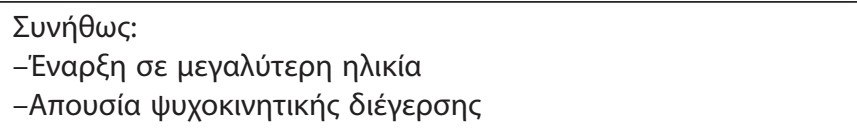 \\
\hline 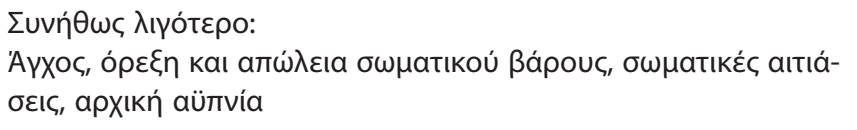 & 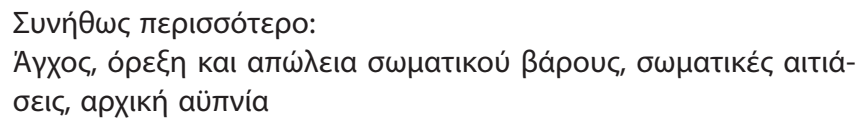 \\
\hline 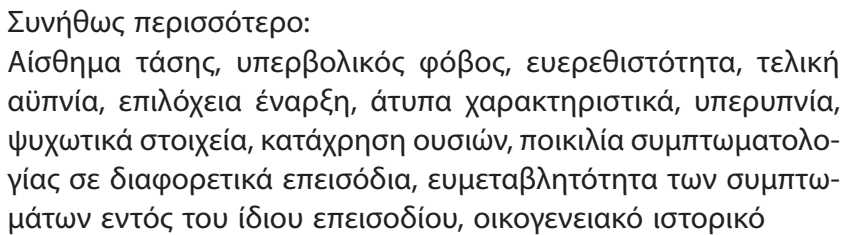 & 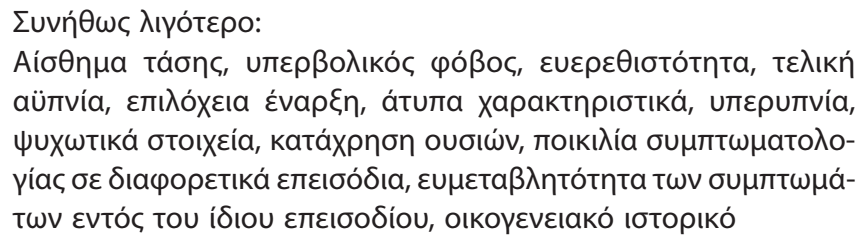 \\
\hline
\end{tabular}

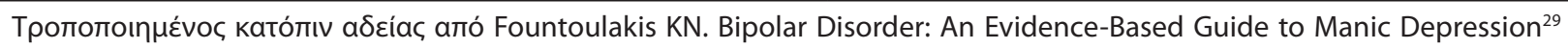




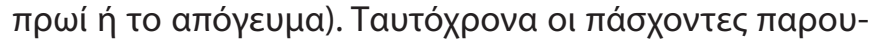

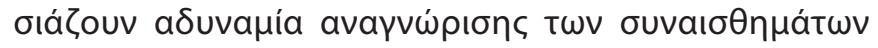

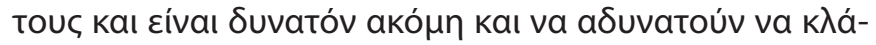

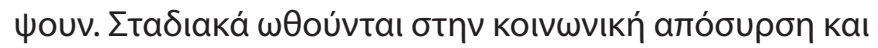

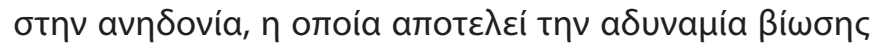

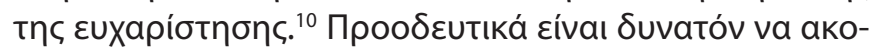

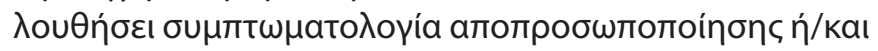

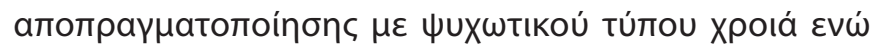

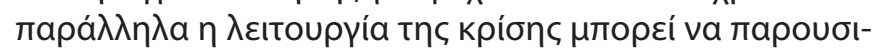

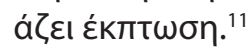

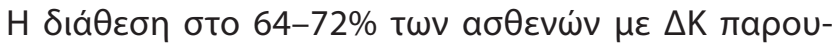

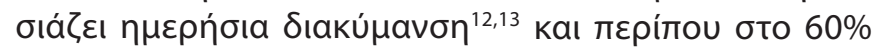

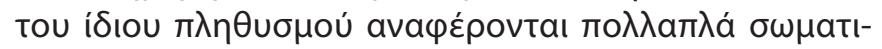

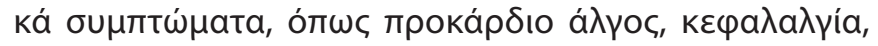

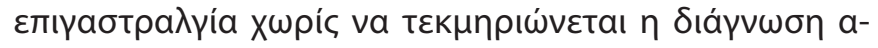

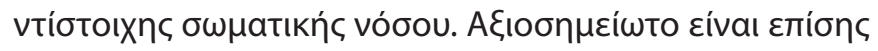

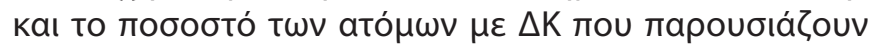

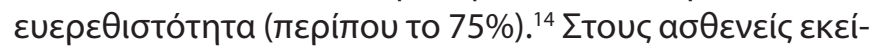

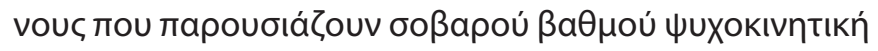

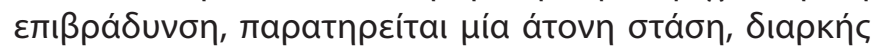

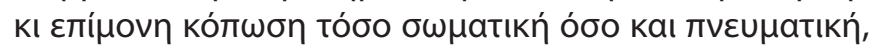

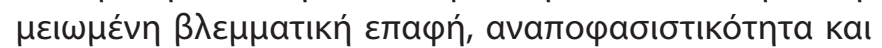

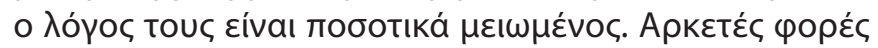

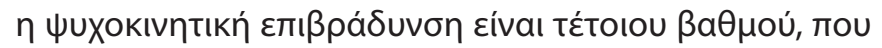

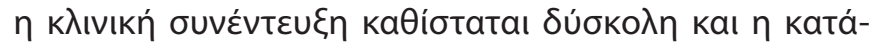

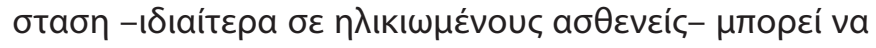

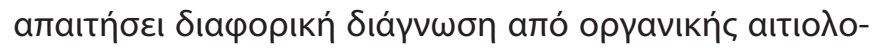

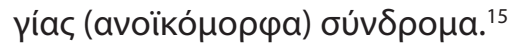

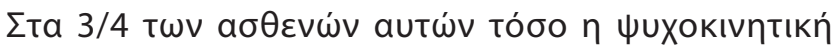

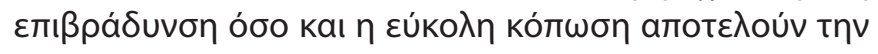

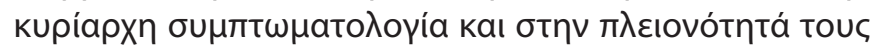

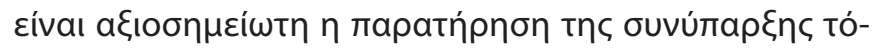

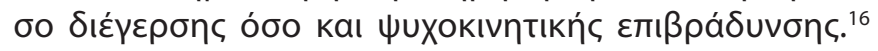

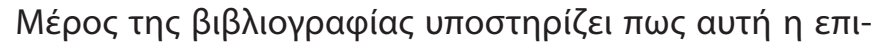

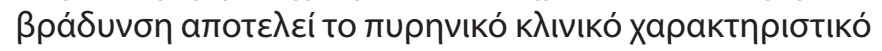

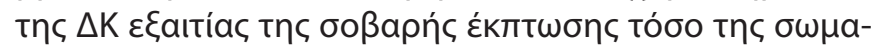

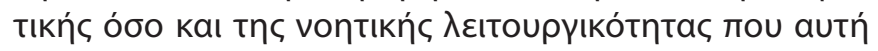

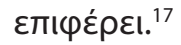

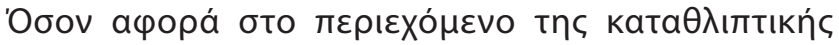

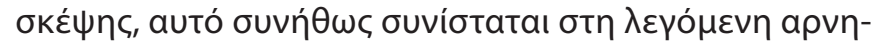

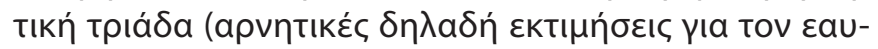

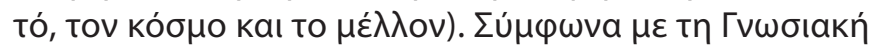

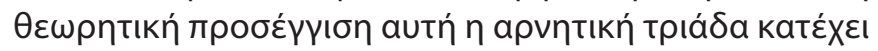

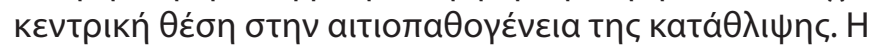

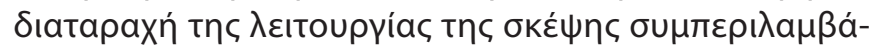

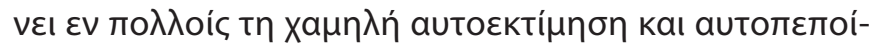

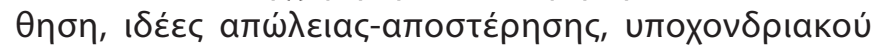

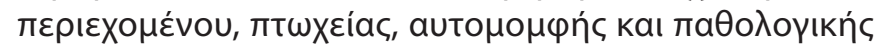

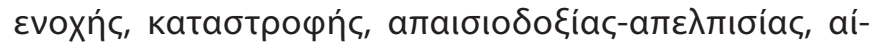

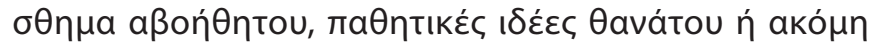

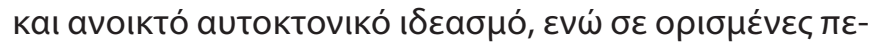

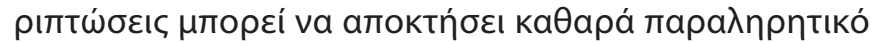
характи́ра. ${ }^{18}$

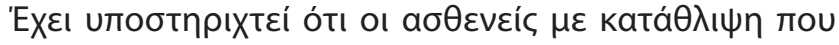

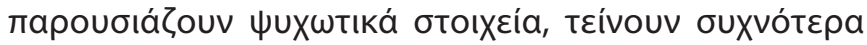

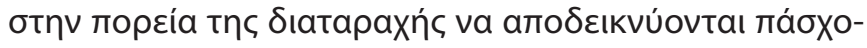

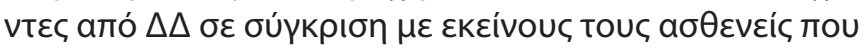

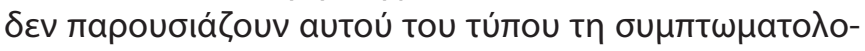

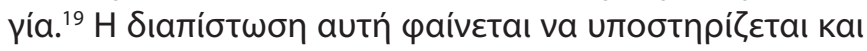

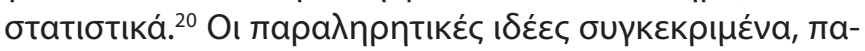

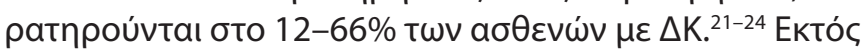

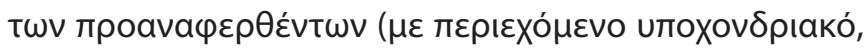

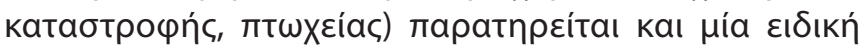

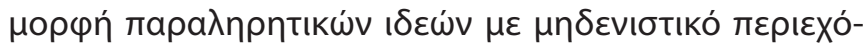

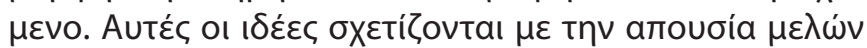

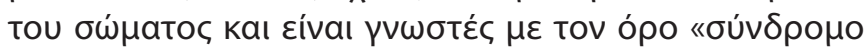
Cotard» (апó to óvoua tou Jules Cotard, 1840-1889, o

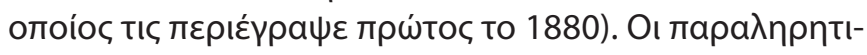

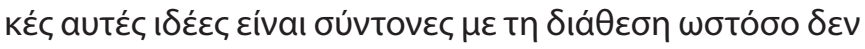

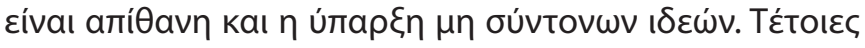

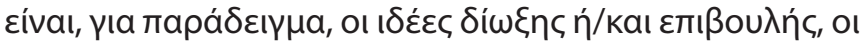

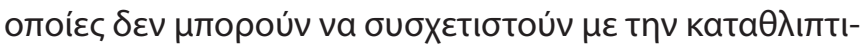

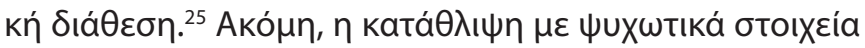

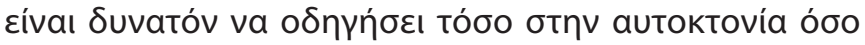

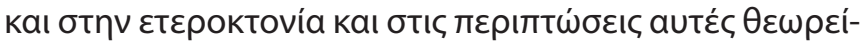

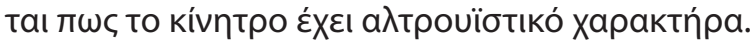

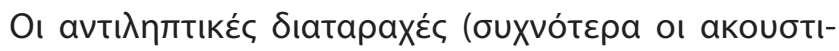

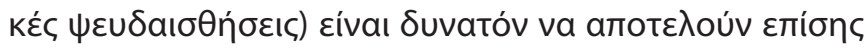

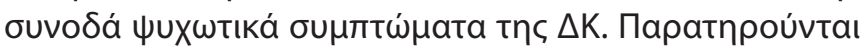

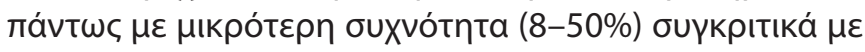

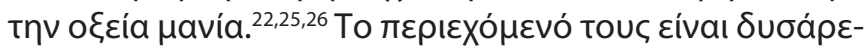

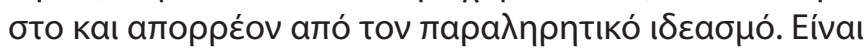

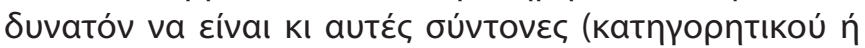

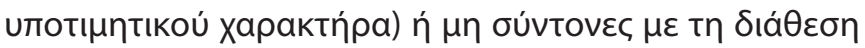

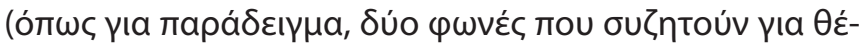

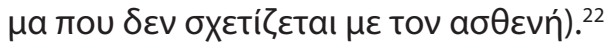

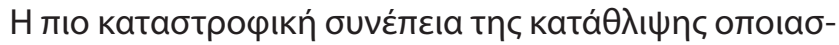

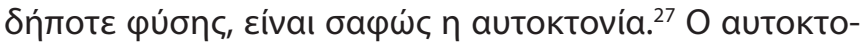

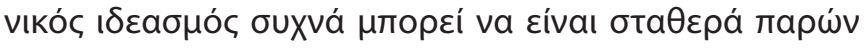

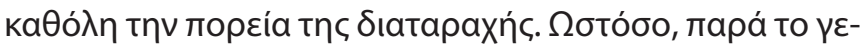

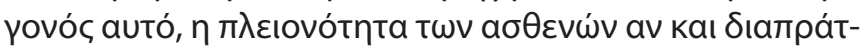

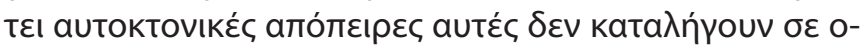

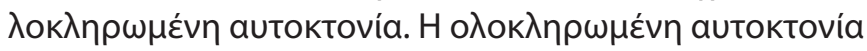

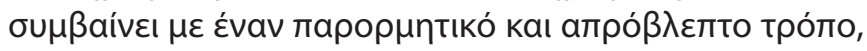

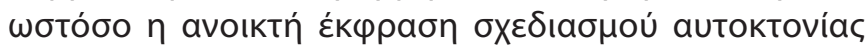

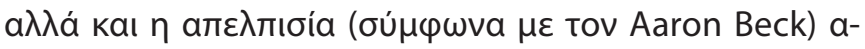

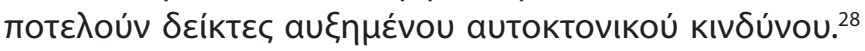

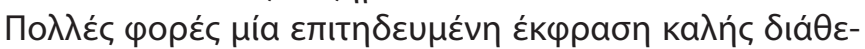

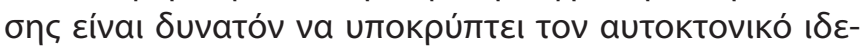

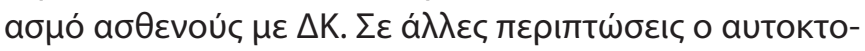

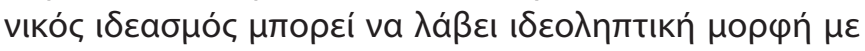

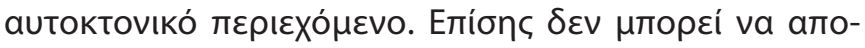

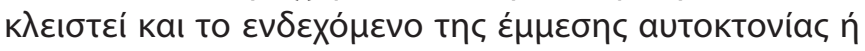

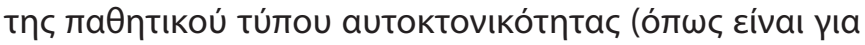

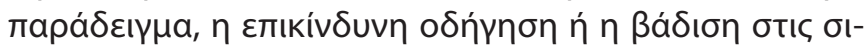

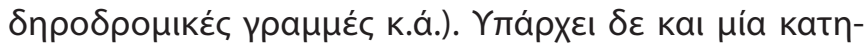


үорі́a катаӨ

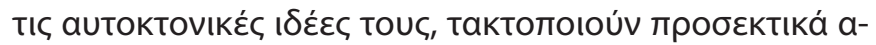

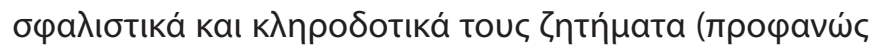

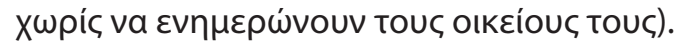

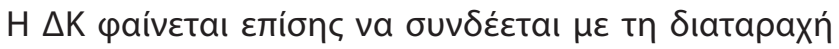

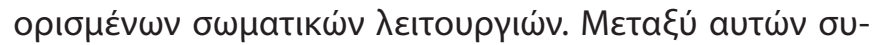

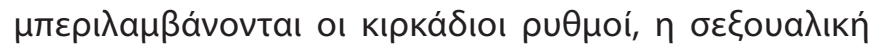

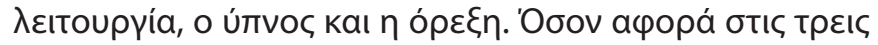

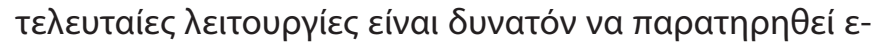

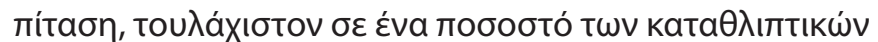

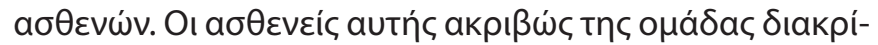

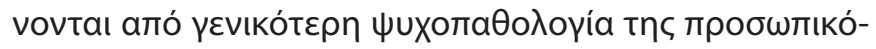

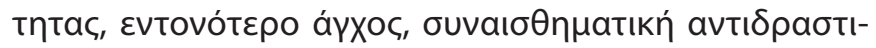

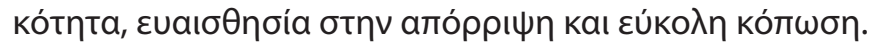

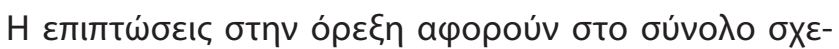

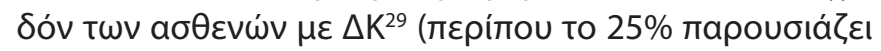

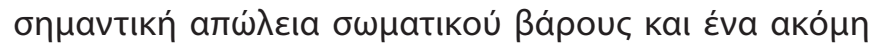

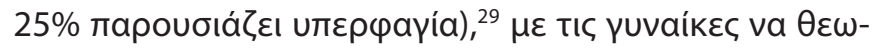

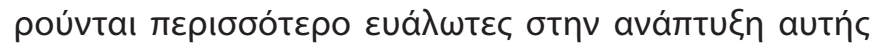

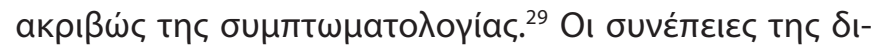

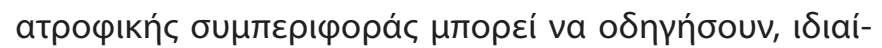

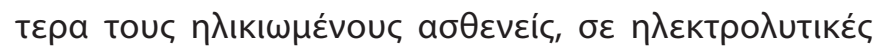

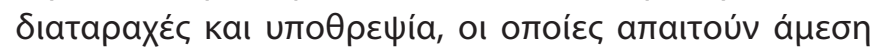

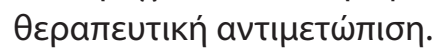

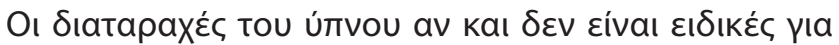

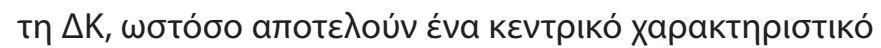

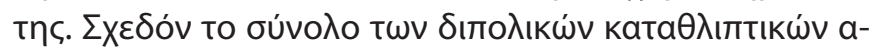

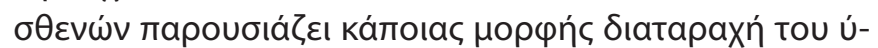

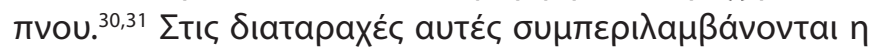

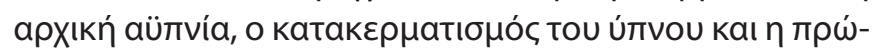

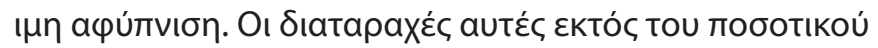

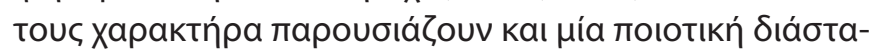

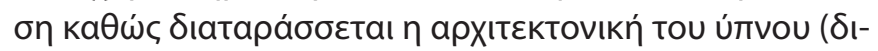

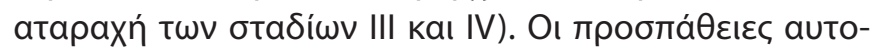

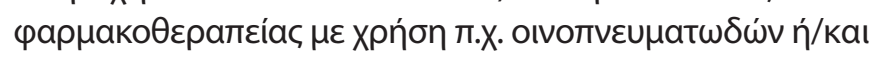

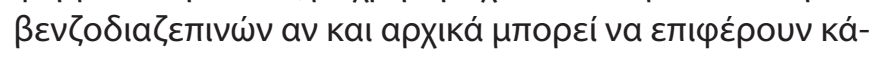

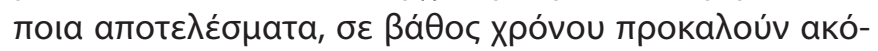

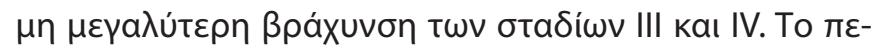

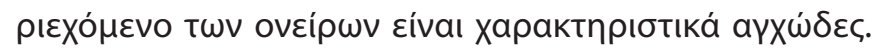

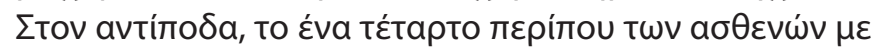

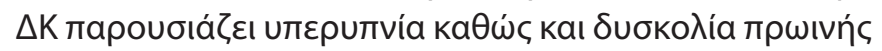

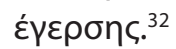

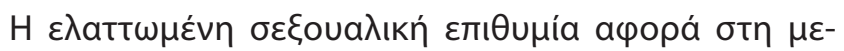

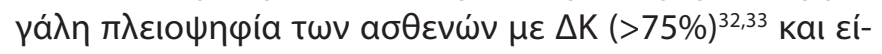

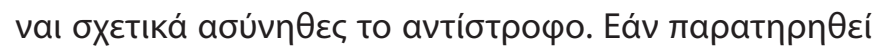

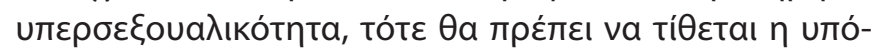

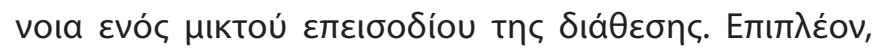

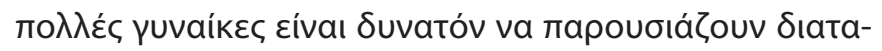

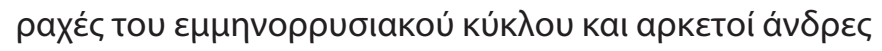

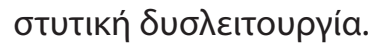

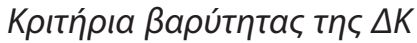

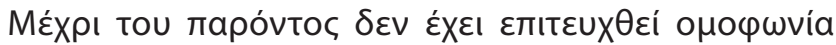

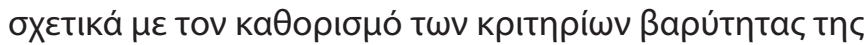

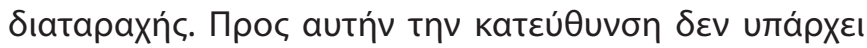

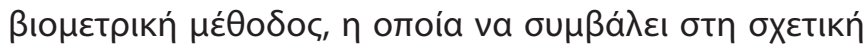

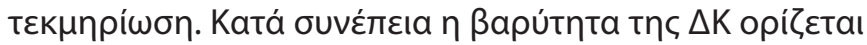

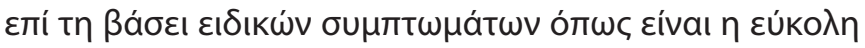

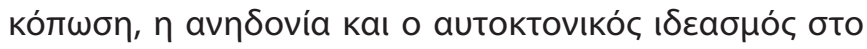

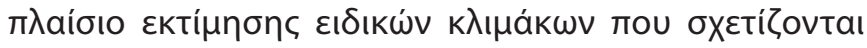

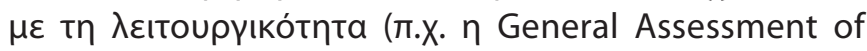
Functioning Scale, GAF). ${ }^{34}$

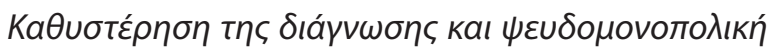 катá $\theta \lambda ı \psi \eta$}

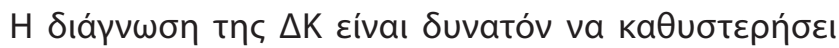

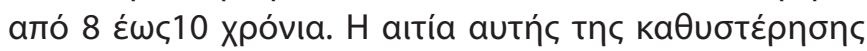

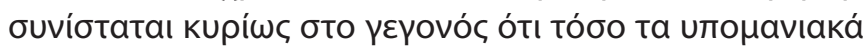

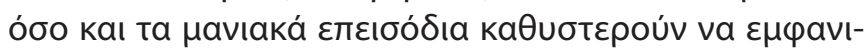

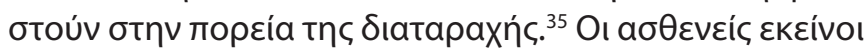

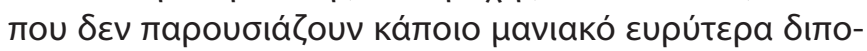

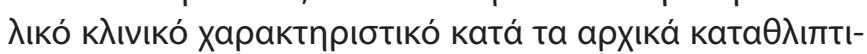

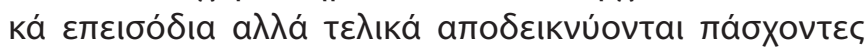

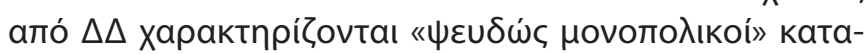

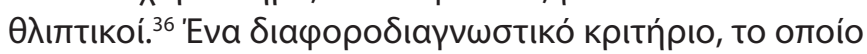

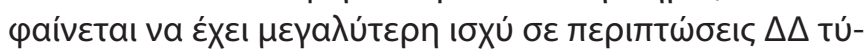

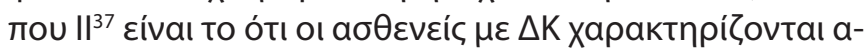

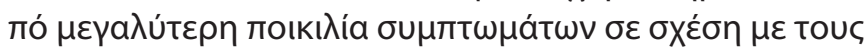

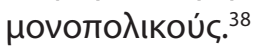

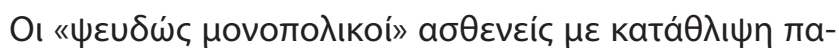

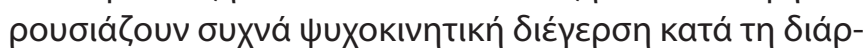

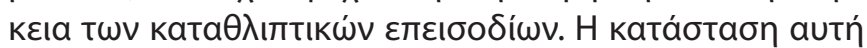

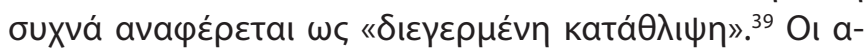

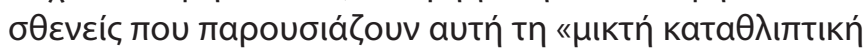

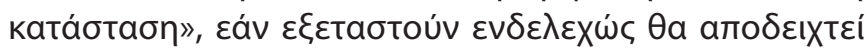

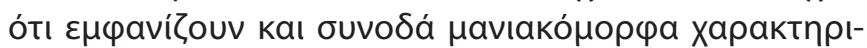

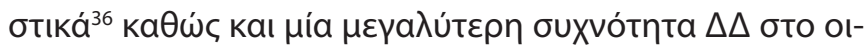

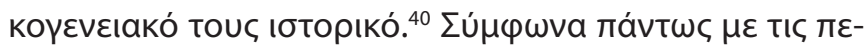

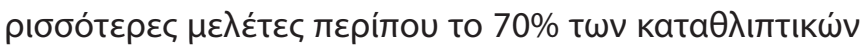

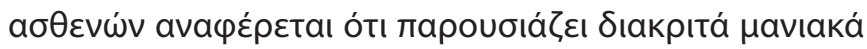

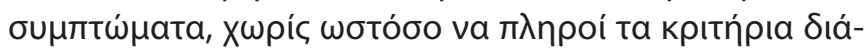

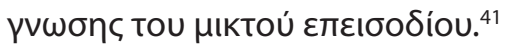

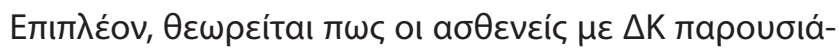

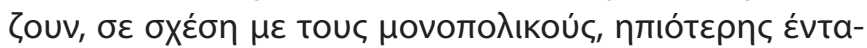

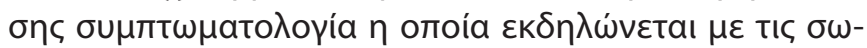

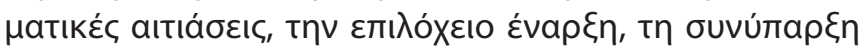

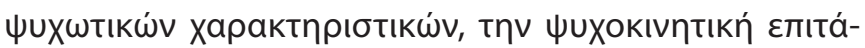

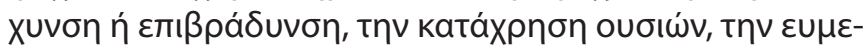

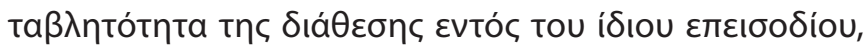

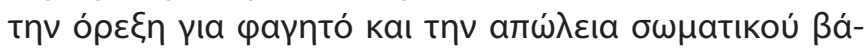

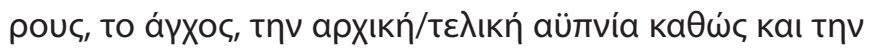
итгритvía каı та átuта характпрıбтıка́. ${ }^{38}$ 


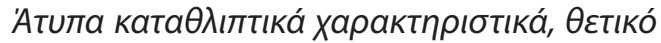

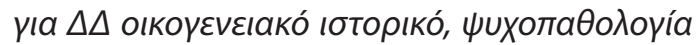

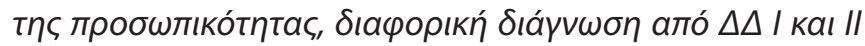

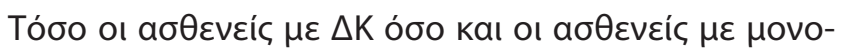

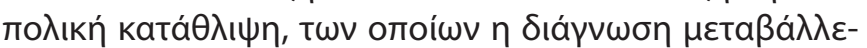

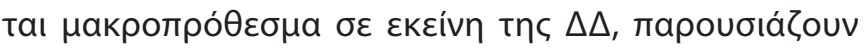

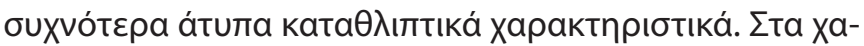

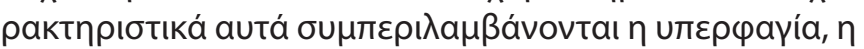

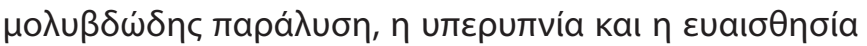

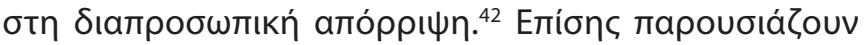

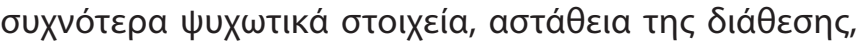

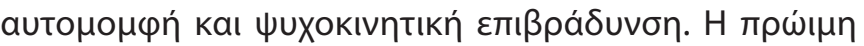

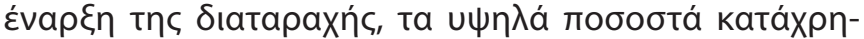

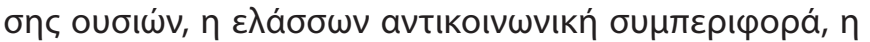

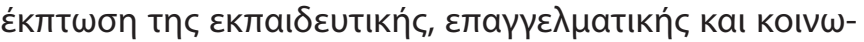

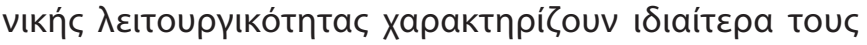

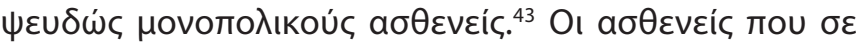

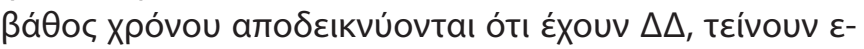

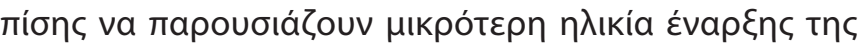

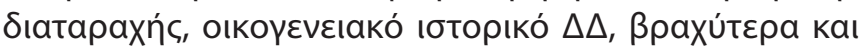

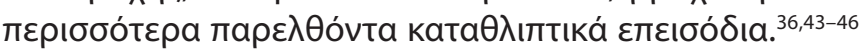

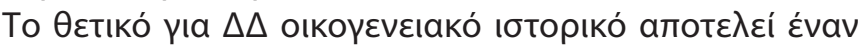

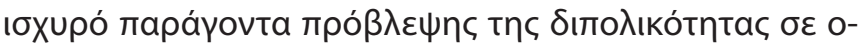

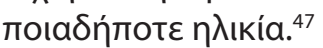

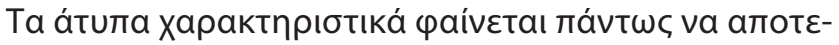

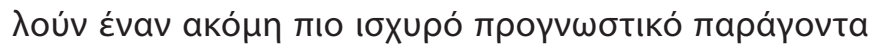
Kal $\varepsilon v \delta \varepsilon X 0 \mu \varepsilon ́ v \omega \varsigma$ a

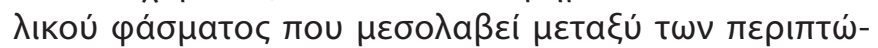

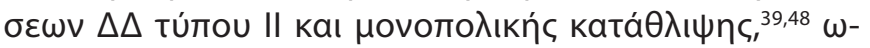

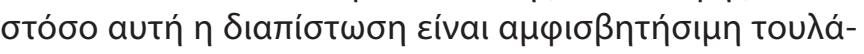

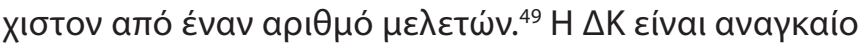

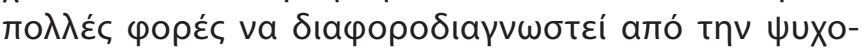

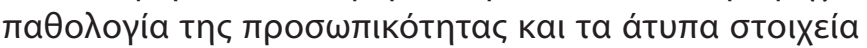

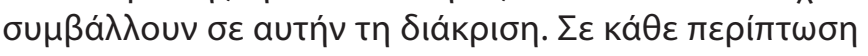

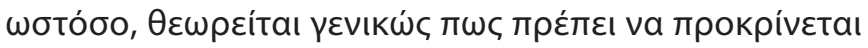

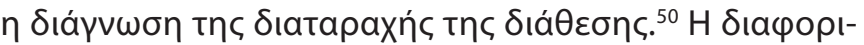

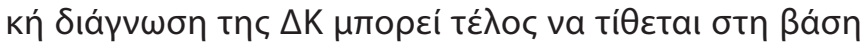

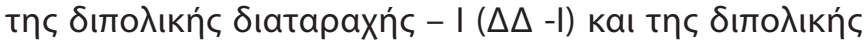

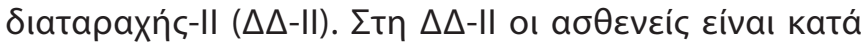

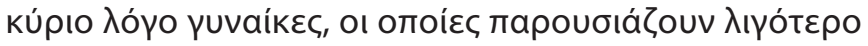

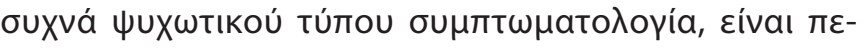

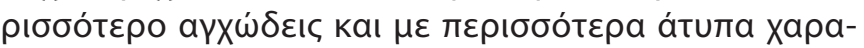

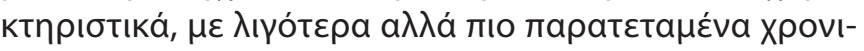

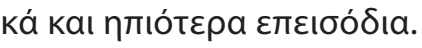

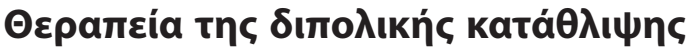

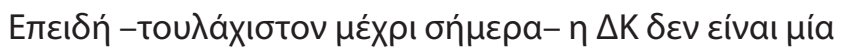

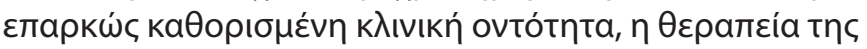

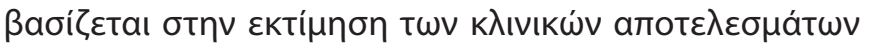

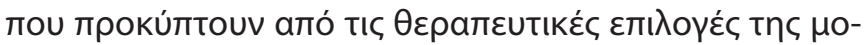

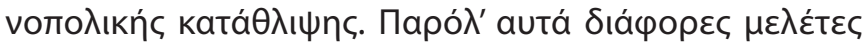

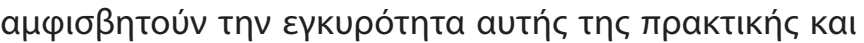

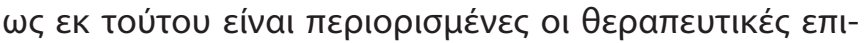

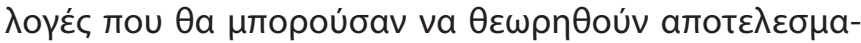

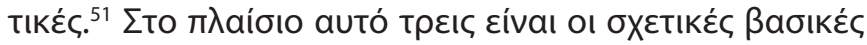

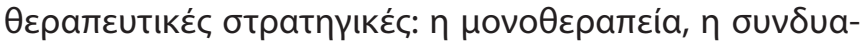

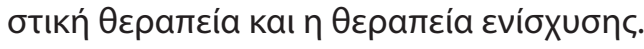

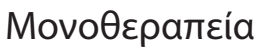

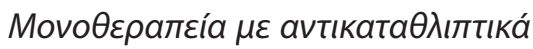

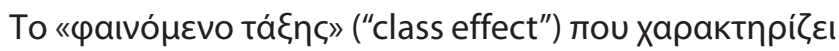

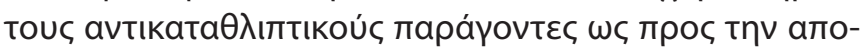

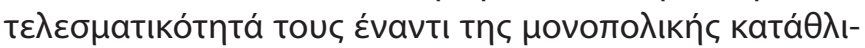

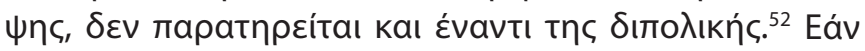

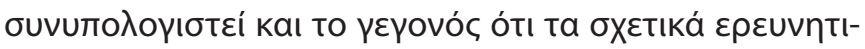

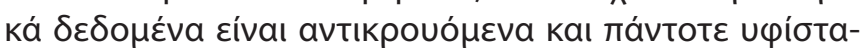

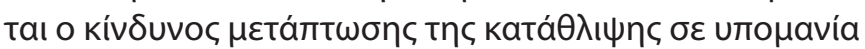

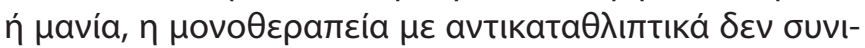

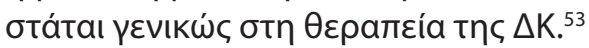

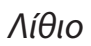

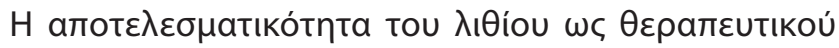

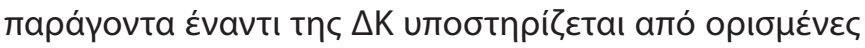

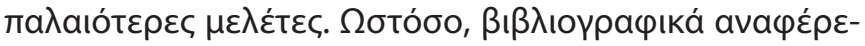

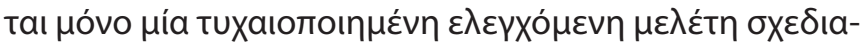

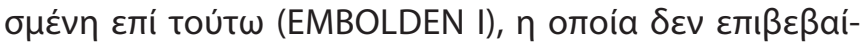

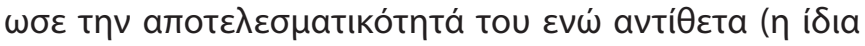

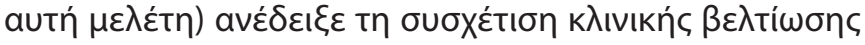

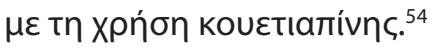

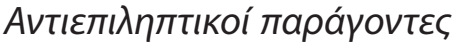 \\ Ва入троїко́}

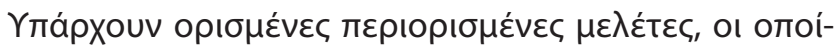

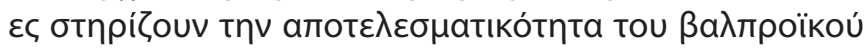

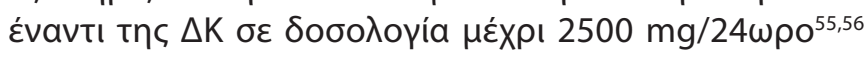

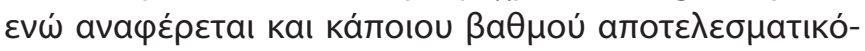

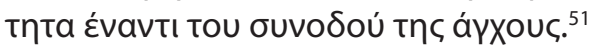

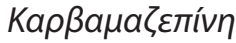

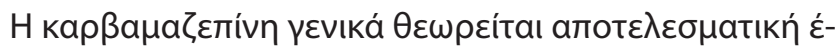

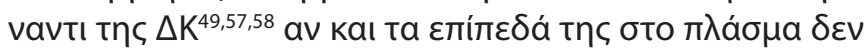

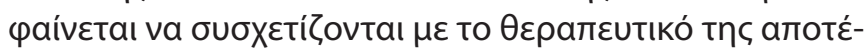
$\lambda \varepsilon \sigma \mu \mathrm{a}^{57}$

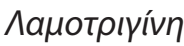

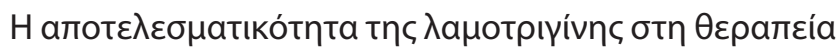

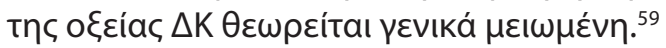

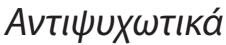

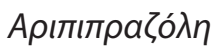

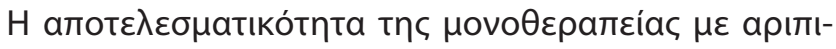

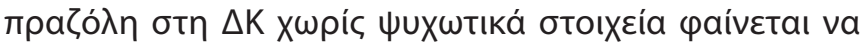

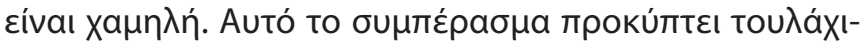

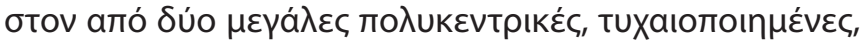

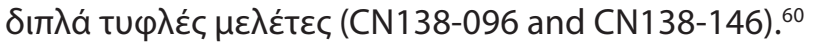




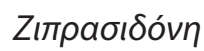

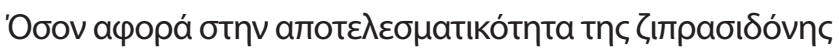

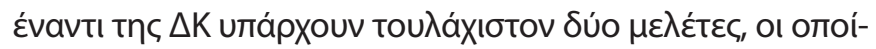

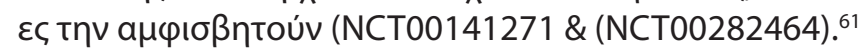

\section{Kovetıativn}

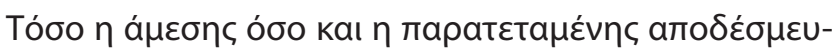

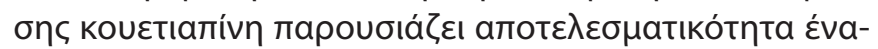

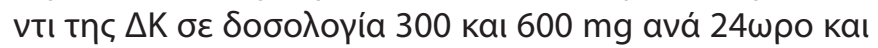

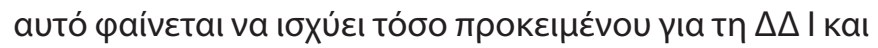

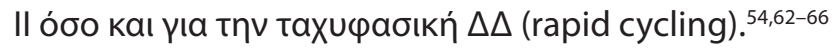

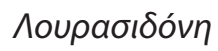

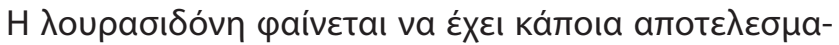

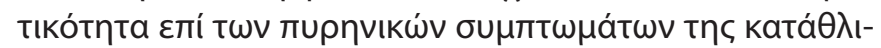

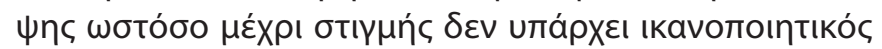

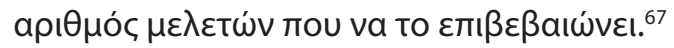

\section{OגavלaTívn}

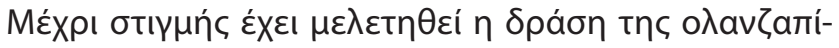

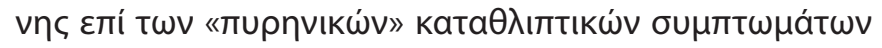

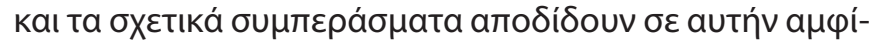

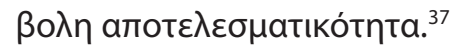

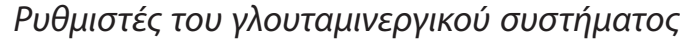

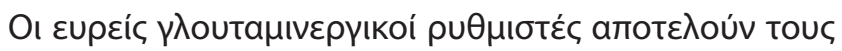

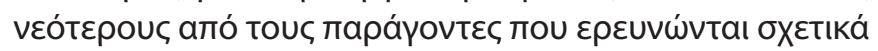
$\mu \varepsilon$ тІৎ avtıKataӨ

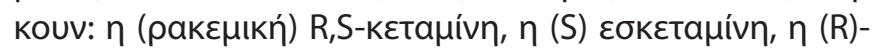

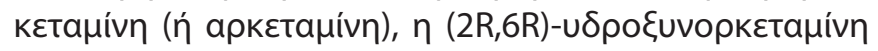

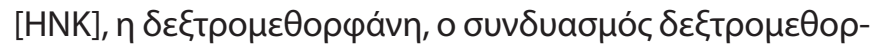

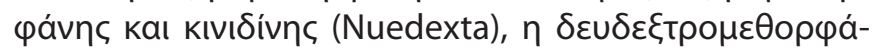

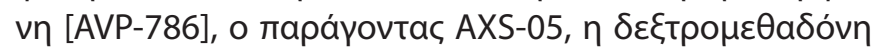

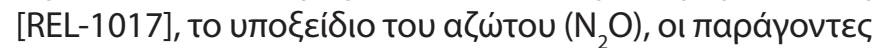

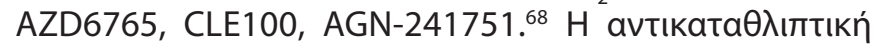

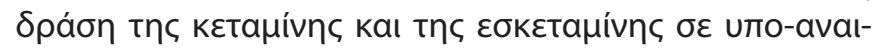

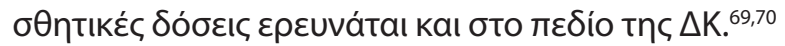

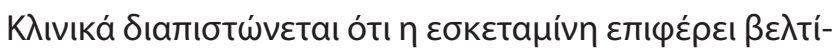

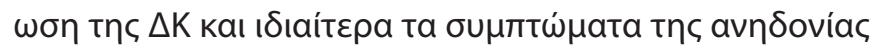

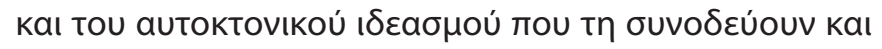

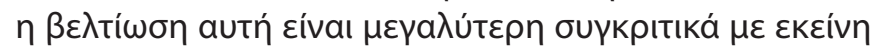

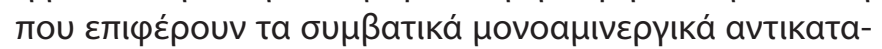

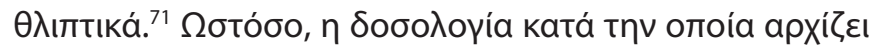

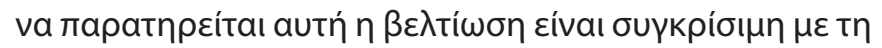

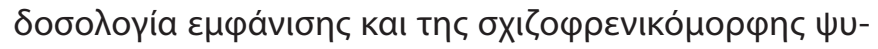

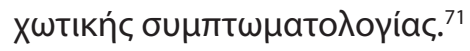

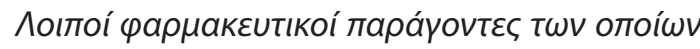

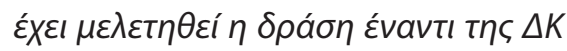

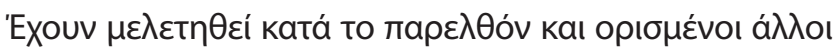

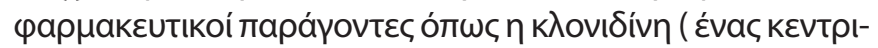

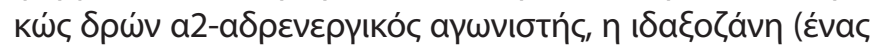

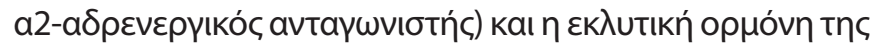

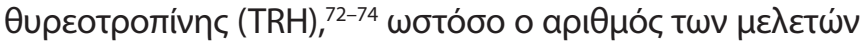

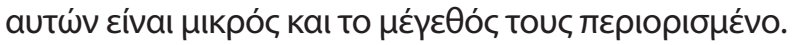

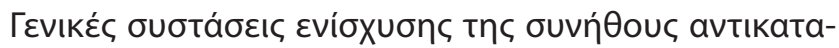

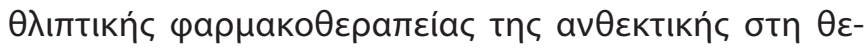

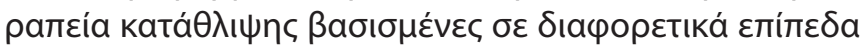

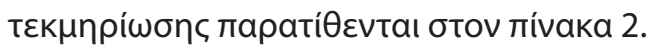

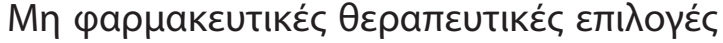

Н

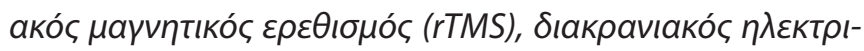

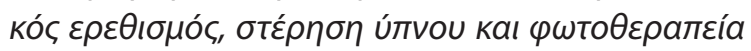

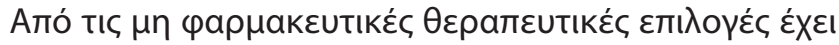

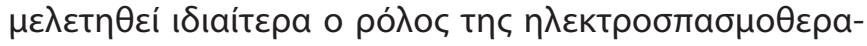

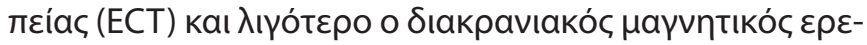

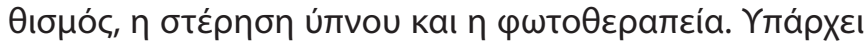

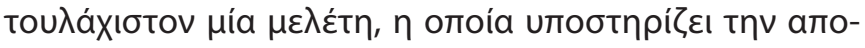

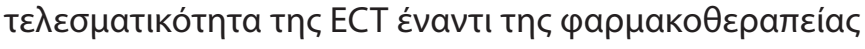

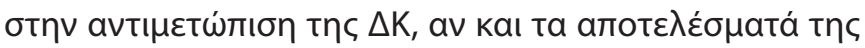

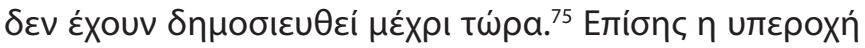

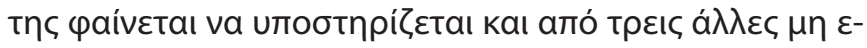

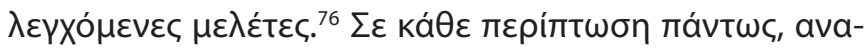

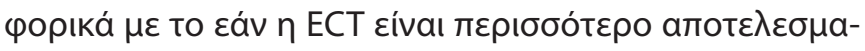

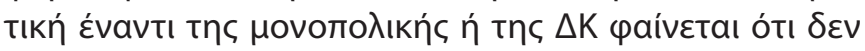

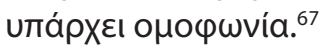

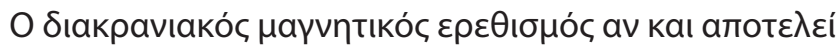
$\mu$ úa $\varepsilon \lambda \pi$

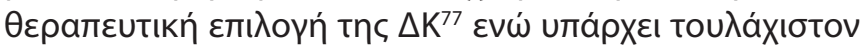

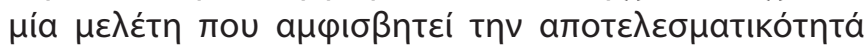

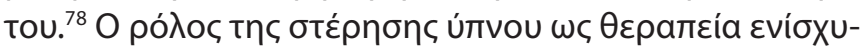

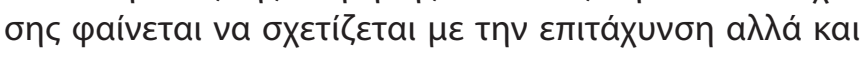

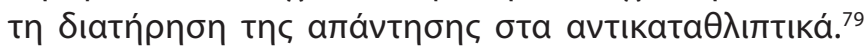

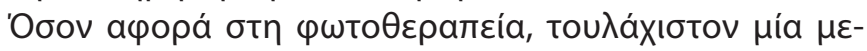

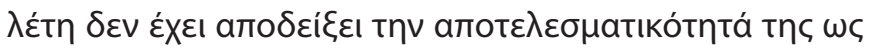

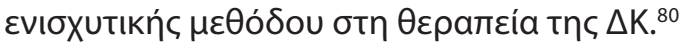

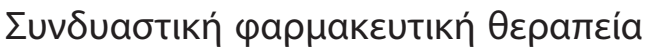

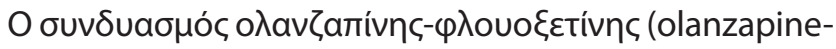

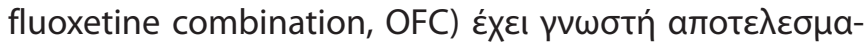

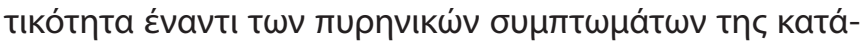

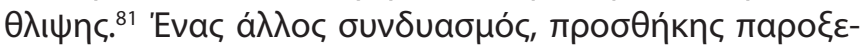

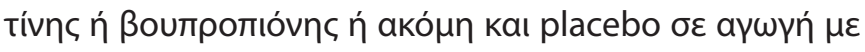

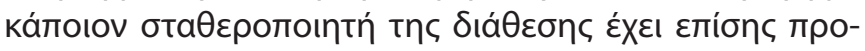

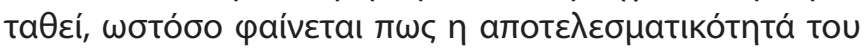

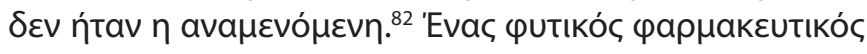

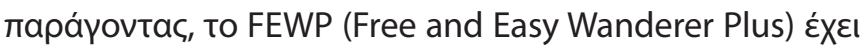

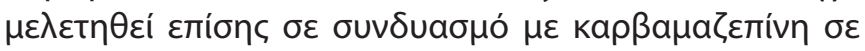

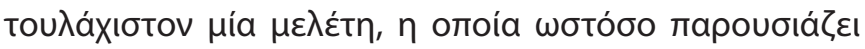

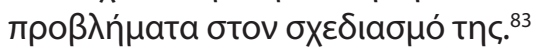

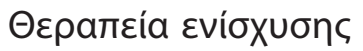

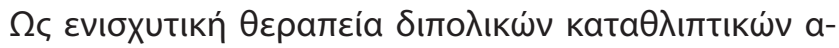

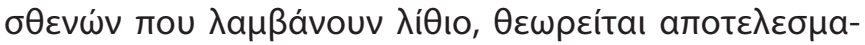




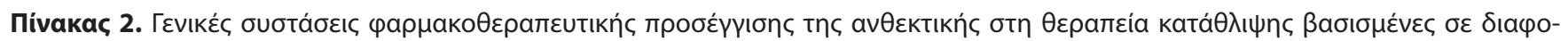

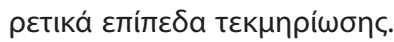

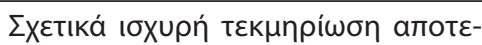

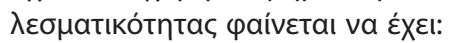

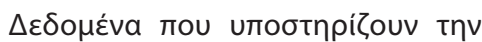

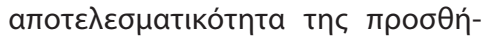

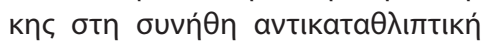

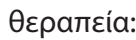

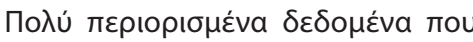

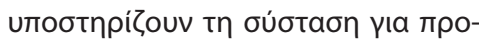

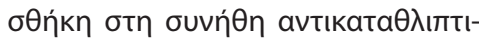

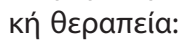

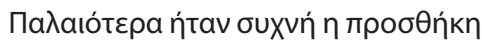

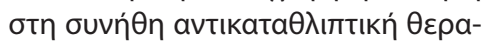
пвía:

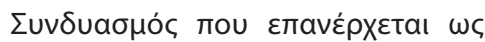

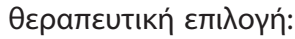

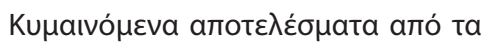

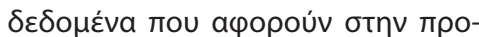

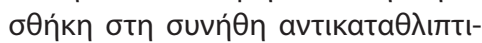

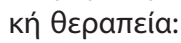

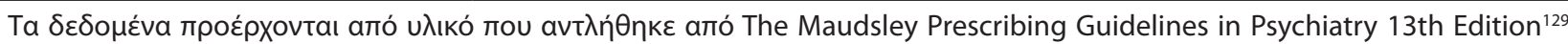

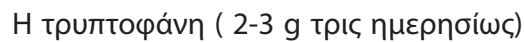

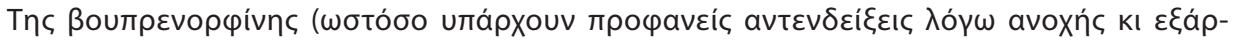

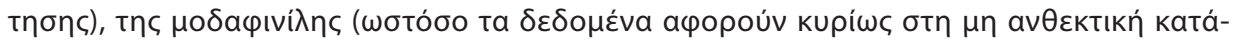

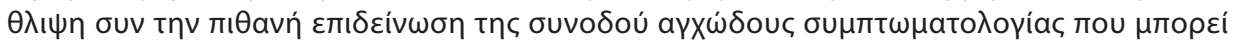

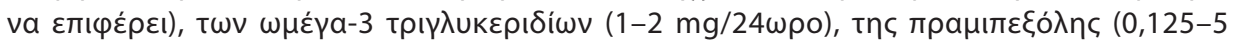

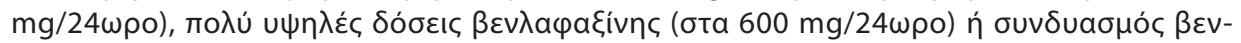

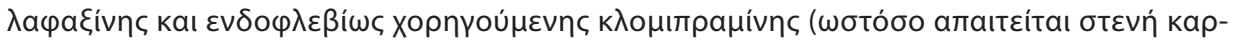

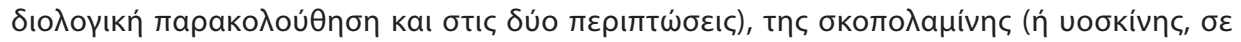

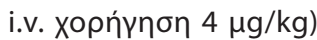

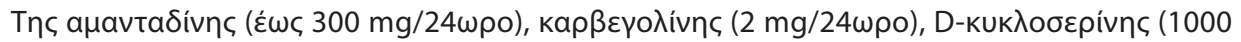

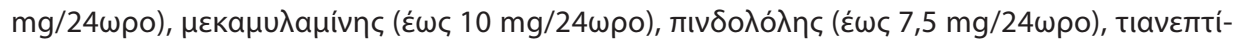

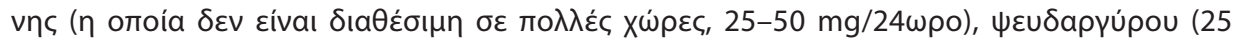

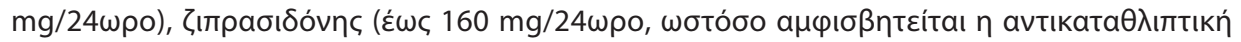

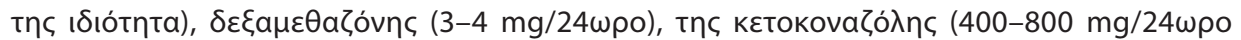

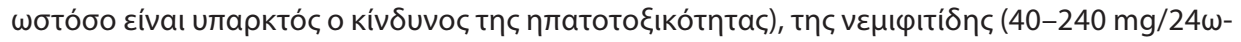

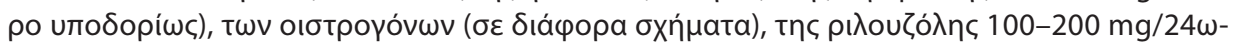

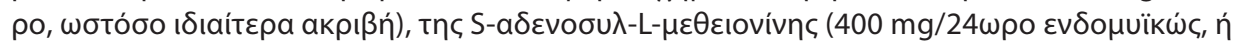

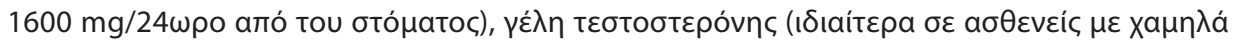

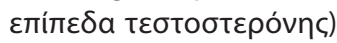

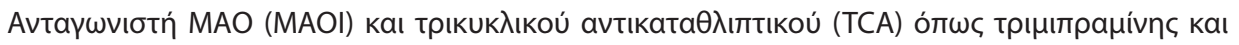

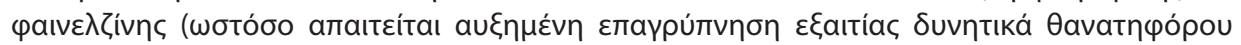

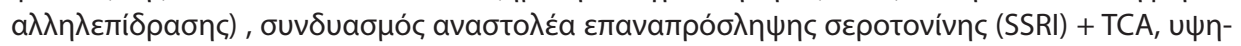

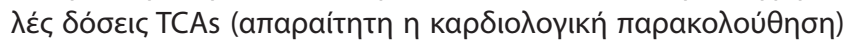

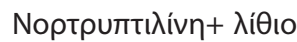

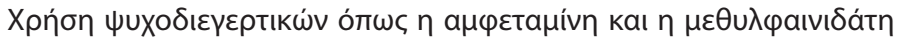

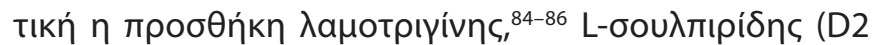

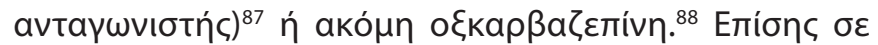

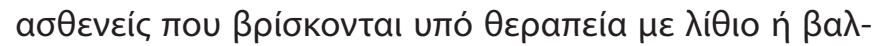

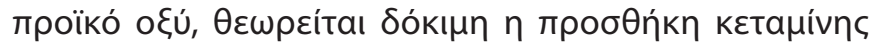

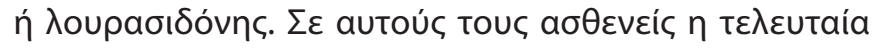

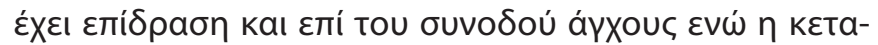

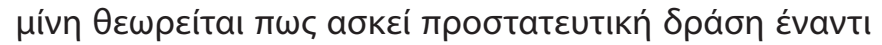

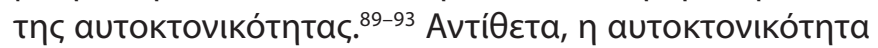

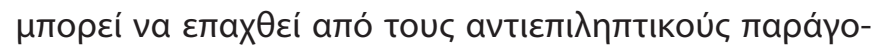

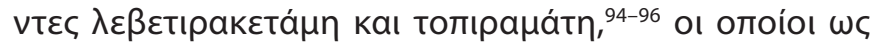

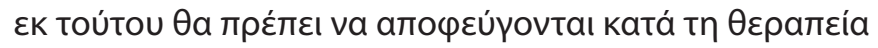

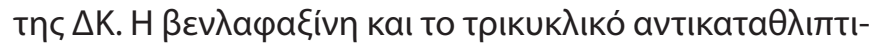

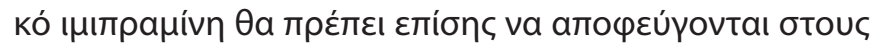

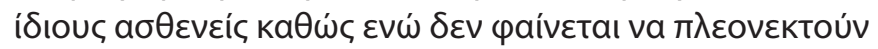

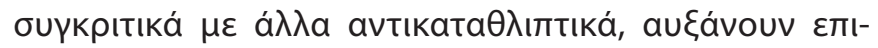

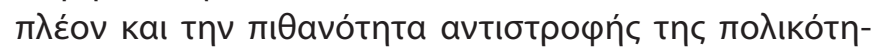
Tac. $^{97-104}$

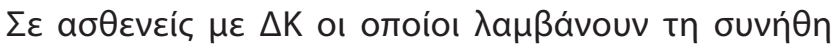

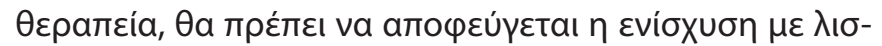

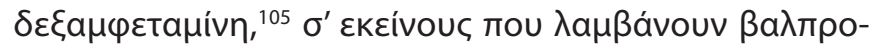

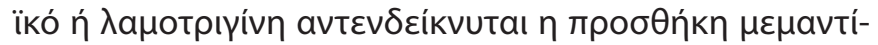

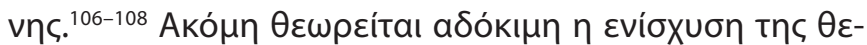

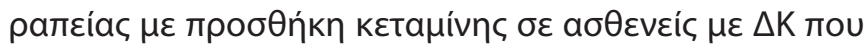

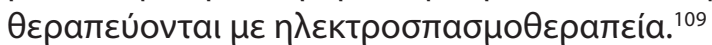

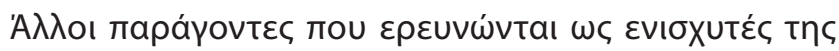

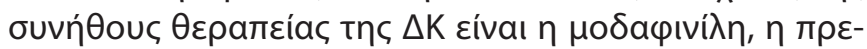

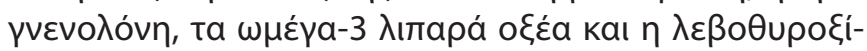

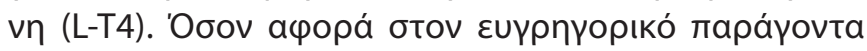

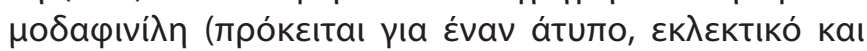

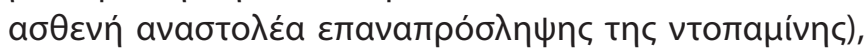

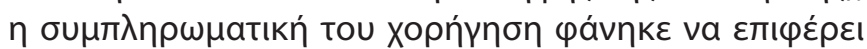

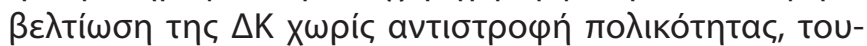

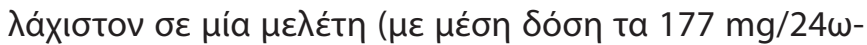

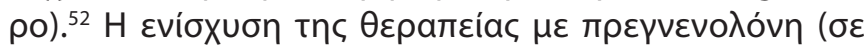

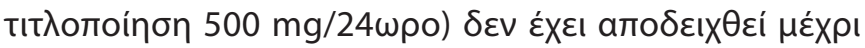

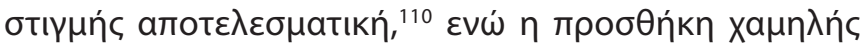

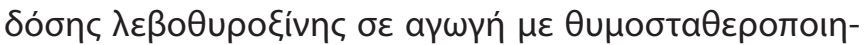

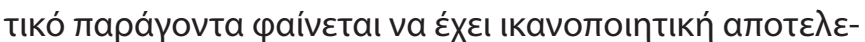

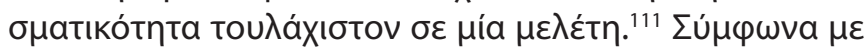

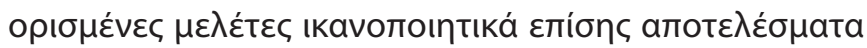

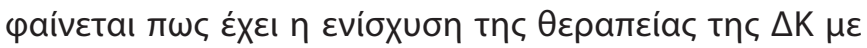

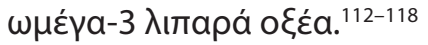




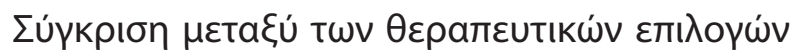

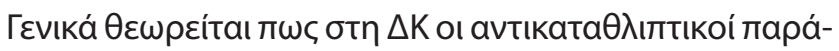

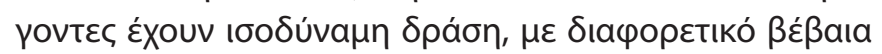

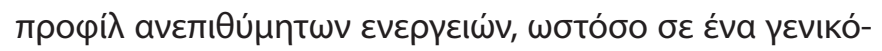

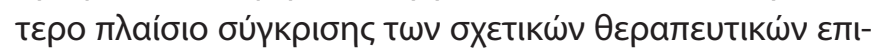

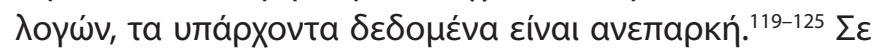

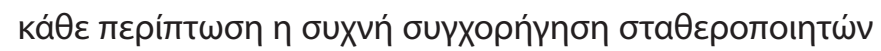

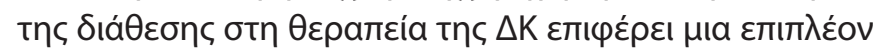

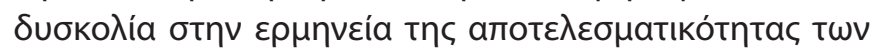

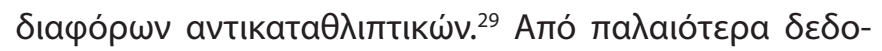

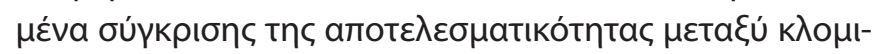

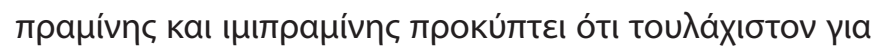

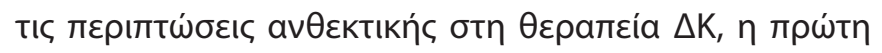

\section{BıBลtoypacpía}

1. Liberati A, Altman DG, Tetzlaff J, Mulrow C, Gøtzsche PC, loannidis JP et al. The PRISMA statement for reporting systematic reviews and meta-analyses of studies that evaluate healthcare interventions: explanation and elaboration. BMJ 2009, 339: b2700, doi: 10.1136/bmj.b2700

2. Moher D, Liberati A, Tetzlaff J, Altman DG, PRISMA Group. Preferred reporting items for systematic reviews and meta-analyses: the PRISMA statement. BMJ 2009, 339:b2535, doi: 10.1136/bmj.b2535

3. Hopewell S, Clarke M, Moher D, Wager E, Middleton P, Altman DG et al. CONSORT for reporting randomized controlled trials in journal and conference abstracts: explanation and elaboration. PLoS Med 2008, 5:e20, doi: 10.1371/journal.pmed.0050020

4. Zhang $\mathrm{K}$ and Hashimoto $\mathrm{K}$. An update on ketamine and its two enantiomers as rapid-acting antidepressants. Expert Rev Neurother 2019, 19:83-92, doi: 10.1080/14737175.2019.1554434

5. Hashimoto K. Rapid-acting antidepressant ketamine, its metabolites and other candidates: A historical overview and future perspective. Psychiatry Clin Neurosci 2019, 73:613-627, doi: 10.1111/pcn.12902

6. Skriptshak $C$ and Reich A. Intranasal esketamine use in bipolar disorder: A case report. Ment Health Clin 2021, 11:259-262, doi: 10.9740/ mhc.2021.07.259

7. Bech P. The full story of lithium. A tribute to Mogens Schou (1918-2005). Psychother Psychosom 2006, 75: 265-269, doi: 10.1159/000093947

8. Davis JM, Janicak PG, Hogan DM. Mood stabilizers in the prevention of recurrent affective disorders: a meta-analysis. Acta Psychiatr Scand 1999, 100:406-417, doi: 10.1111/j.1600-0447.199.tb10890.x

9. Burgess S, Geddes J, Hawton K, Townsend E, Jamison K, Goodwin G. Lithium for maintenance treatment of mood disorders. Cochrane Database Syst Rev 2001: CD003013, doi: 10.1002/14651858.CD003013

10. Gijsman HJ, Geddes JR, Rendell JM, Nolen WA, Goodwin GM. Antidepressants for bipolar depression: a systematic review of randomized, controlled trials. Am J Psychiatry 2004, 161:1537-1547, doi: 10.1176/ appi.ajp.161.9.1537

11. Rendell JM, Gijsman HJ, Bauer MS, Goodwin GM, Geddes GR. Risperidone alone or in combination for acute mania. Cochrane Database Syst Rev 2006, 2006:CD004043, doi: 10.1002/14651858.CD004043.pub2

12. Macritchie K, Geddes JR, Scott J, Haslam D, de Lima M, Goodwin G. Valproate for acute mood episodes in bipolar disorder. Cochrane Database Syst Rev 2003, 2003:CD004052, doi: 10.1002/14651858.CD004052

13. Rendell JM, Gijsman HJ, Keck P, Goodwin GM, Geddes JR. Olanzapine alone or in combination for acute mania. Cochrane Database Syst Rev 2003, 2003:CD004040, doi: 10.1002/14651858.CD004040

14. Macritchie KA, Geddes JR, Scott J, Haslam DR, Goodwin GM. Valproic acid, valproate and divalproex in the maintenance treatment of bipolar disorder. Cochrane Database Syst Rev 2001, 2001:CD003196, doi: 10.1002/14651858.CD003196

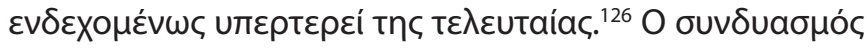

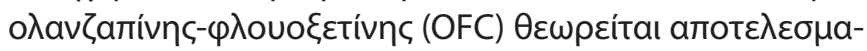

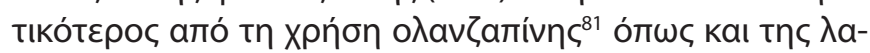

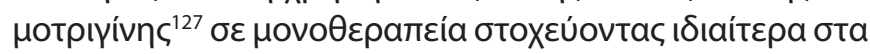

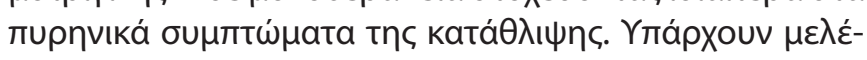

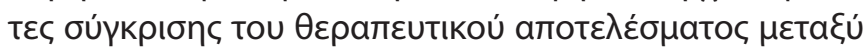

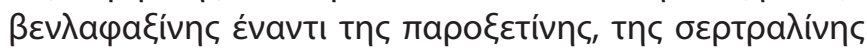

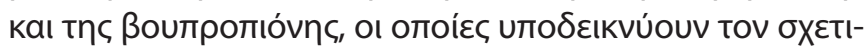

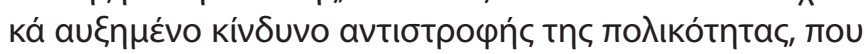

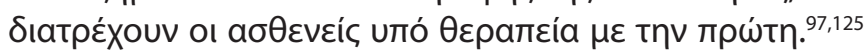

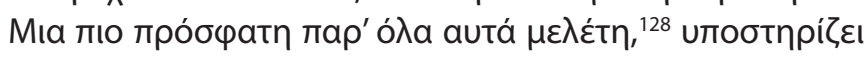

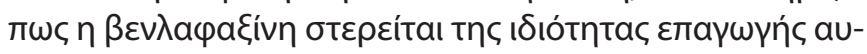

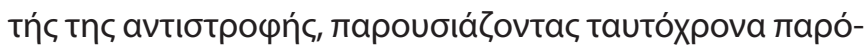

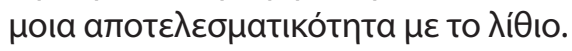

15. Cipriani A, Smith K, Burgess S, Carney S, Goodwin G, Geddes J. Lithium versus antidepressants in the long-term treatment of unipolar affective disorder. Cochrane Database Syst Rev 2006, 2006:CD003492, doi: 10.1002/14651858.CD003492.pub2

16. Cipriani A, Rendell JM, Geddes JR. Haloperidol alone or in combination for acute mania. Cochrane Database Syst Rev 2006, 2006:CD004362, doi: 10.1002/14651858.CD004362.pub2

17. Smith LA, Cornelius V, Warnock A, Tacchi MJ, Taylor D. Acute bipolar mania: a systematic review and meta-analysis of co-therapy vs. monotherapy. Acta Psychiatr Scand 2007, 115:12-20, doi: 10.1111/j.16000447.2006.00912.x

18. Gao K, Gajwani P, Elhaj O, Calabrese JR. Typical and atypical antipsychotics in bipolar depression. J Clin Psychiatry 2005, 66:1376-1385, doi: 10.4088/jcp.v66n1106

19. Nivoli AM, Murru A, Goikolea JM, Crespo JM, Montes JM, González-Pinto $A$ et al. New treatment guidelines for acute bipolar mania: a critical review. J Affect Disord 2012, 140:125-141, doi: 10.1016/j.jad.2011.10.015

20. Nivoli AM, Colom F, Murru A, Pacchiarotti I, Castro-Loli P, González-Pinto A et al. New treatment guidelines for acute bipolar depression: a systematic review. J Affect Disord 2011, 129:14-26, doi: 10.1016/j.jad.2010.05.018

21. Cipriani A, Barbui C, Salanti G, Rendell J, Brown R, Stockton S et al. Comparative efficacy and acceptability of antimanic drugs in acute mania: a multiple-treatments meta-analysis. Lancet 2011, 378:1306-1315, doi: 10.1016/S0140-6736(11)60873-8

22. Fountoulakis KN, VietaE. Treatment of bipolar disorder:a systematic review of available data and clinical perspectives. Int J Neuropsychopharmacol 2008, 11:999-1029, doi: 10.1017/S146114570 8009231

23. Tarr GP, Herbison P, de la Barra SL, Glue P. Study design and patient characteristics and outcome in acute mania clinical trials. Bipolar Disord 2011, 13:125-132, doi: 10.1111/j.1399-5618.2011.00904.x

24. Yildiz A, Vieta E, Leucht S, Baldessarini RJ. Efficacy of antimanic treatments: meta-analysis of randomized, controlled trials. Neuropsychopharmacology 2011, 36:375-389, doi: 10.1038/npp.2010.192

25. Fountoulakis KN, Kasper S, Andreassen O, Blier P, Okasha A, Severus E et al. Efficacy of pharmacotherapy in bipolar disorder: a report by the WPA section on pharmacopsychiatry. Eur Arch Psychiatry Clin Neurosci 2012, 262(Suppl 1):1-48, doi: 10.1007/s00406-012-0323-x

26. Fountoulakis KN, Vieta E, Sanchez-Moreno J, Kaprinis SG, Goikolea JM, Kaprinis GS. Treatment guidelines for bipolar disorder: a critical review. J Affect Disord 2005, 86:1-10, doi: 10.1016/j.jad.2005.01.004

27. Yatham LN, Kusumakar V, Parikh SV, Haslam DR, Matte R, Sharma V et al. Bipolar depression: treatment options. Can J Psychiatry 1997, 42(Suppl 2):87S-91S, PMID: 9288441

28. Srisurapanont M, Yatham LN, Zis AP. Treatment of acute bipolar depression: a review of the literature. Can J Psychiatry 1995, 40:533-544, doi: $10.1177 / 070674379504000906$ 
29. Fountoulakis KN. Bipolar Disorder: An Evidence-Based Guide to Manic Depression. Springer, Berlin Heidelberg, 2015

30. Fountoulakis KN. The contemporary face of bipolar illness: complex diagnostic and therapeutic challenges. CNS Spectr 2008, 13:763-774, 777-769, doi: 10.1017/s1092852900013894

31. Fountoulakis KN. Refractoriness in bipolar disorder: definitions and evidence-based treatment. CNS Neurosci Ther 2012, 18:227-237, doi: 10.1111/j.1755-5949.2011.00259.x

32. Fountoulakis KN, Giannakopoulos P, Kövari E, Bouras C. Assessing the role of cingulate cortex in bipolar disorder: neuropathological, structural and functional imaging data. Brain Res Rev 2008, 59:9-21, doi: 10.1016/j. brainresrev.2008.04.005

33. Fountoulakis KN, Gonda X, Siamouli M, Rihmer Z. Psychotherapeutic intervention and suicide risk reduction in bipolar disorder: a review of the evidence. J Affect Disord 2009, 113:21-29, doi: 10.1016/j.jad.2008. 06.014

34. Stokes PE, Shamoian CA, Stoll PM, Patton MJ. Efficacy of lithium as acute treatment of manic-depressive illness. Lancet 1971, 1:1319-1325, doi: 10.1016/s0140-6736(71)91886-1

35. Keck PE, Orsulak PJ, Cutler AJ, Sanchez R, Torbeyns A, Marcus RN et al. Aripiprazole monotherapy in the treatment of acute bipolar I mania: a randomized, double-blind, placebo- and lithium-controlled study. J Affect Disord 2009, 112:36-49, doi: 10.1016/j.jad.2008.05.014

36. Bowden CL, Brugger AM, Swann AC, Calabrese JR, Janicak PG, Petty $F$ et al. Efficacy of divalproex vs lithium and placebo in the treatment of mania. The Depakote Mania Study Group. JAMA 1994, 271:918-924, PMID: 8120960

37. Kushner SF, Khan A, Lane R, Olson WH. Topiramate monotherapy in the management of acute mania: results of four double-blind placebo-controlled trials. Bipolar Disord 2006, 8:15-27, doi: 10.1111/j.13995618.2006.00276.x

38. Bowden $C L$, Grunze $H$, Mullen J, Brecher $M$, Paulsson $B$, Jones $M$ et al. A randomized, double-blind, placebo-controlled efficacy and safety study of quetiapine or lithium as monotherapy for mania in bipolar disorder. J Clin Psychiatry 2005, 66:111-121, doi: 10.4088/jcp.v66n0116

39. Swann AC, Bowden CL, Morris D, Calabrese JR, Petty F, Small J et al. Depression during mania. Treatment response to lithium or divalproex. Arch Gen Psychiatry 1997, 54:37-42, doi: 10.1001/archpsyc.1997. 01830130041008

40. McElroy SL, Keck PE Jr, Pope HG Jr, Hudson J. Valproate in psychiatric disorders: literature review and clinical guidelines. J Clin Psychiatry 1989, 50(Suppl):23-29, PMID: 2494155

41. Emrich HM, von Zerssen D, Kissling W, Möller HJ, Windorfer A. Effect of sodium valproate on mania. The GABA-hypothesis of affective disorders. Arch Psychiatr Nervenkr 1980, 229:1-16, doi: 10.1007/BF00343800

42. Emrich HM, von Zerssen D, Kissling W, Möller HJ. Therapeutic effect of valproate in mania. Am J Psychiatry 1981, 138:256, doi: 10.1176/ ajp.138.2.256

43. Pope HG Jr, McElroy SL, Keck PE Jr, Hudson JI. Valproate in the treatment of acute mania. A placebo-controlled study. Arch Gen Psychiatry 1991, 48:62-68, doi: 10.1001/archpsyc.1991.01810250064008

44. Bowden CL, Swann AC, Calabrese JR, Rubenfaer LM, Wozniak PJ, Collins $\mathrm{MA}$ et al. A randomized, placebo-controlled, multicenter study of divalproex sodium extended release in the treatment of acute mania. J Clin Psychiatry 2006, 67:1501-1510, doi: 10.4088/jcp.v67n1003

45. Hirschfeld RM, Bowden CL, Vigna NV, Wozniak P, Collins M. A randomized, placebo-controlled, multicenter study of divalproex sodium extended-release in the acute treatment of mania. J Clin Psychiatry 2010, 71:426-432, doi: 10.4088/JCP.08m04960yel

46. Tohen M, Vieta E, Goodwin GM, Sun B, Amsterdam JD, Banov M et al. Olanzapine versus divalproex versus placebo in the treatment of mild to moderate mania: a randomized, 12-week, double-blind study. J Clin Psychiatry 2008, 69:1776-1789, doi: 10.4088/jcp.v69n1113

47. McElroy SL, Martens BE, Creech RS, Welge JA, Jefferson L, Guerdjikova AI et al. Randomized, double-blind, placebo-controlled study of divalproex extended release loading monotherapy in ambulatory bipolar spectrum disorder patients with moderate-to-severe hypomania or mild mania. $J$ Clin Psychiatry 2010, 71:557-565, doi: 10.4088/JCP.08m04854yel

48. Ballenger JC, Post RM. Carbamazepine in manic-depressive illness: a new treatment. Am J Psychiatry 1980, 137:782-790, doi: 10.1176/ajp.137.7.782

49. Post RM, Unde TW, Roy-Byrne PP, Joffe RT. Correlates of antimanic response to carbamazepine. Psychiatry Res 1987, 21:71-83, doi: 10.1016/0165-1781(87)90064-3

50. Weisler RH, Kalali AH, Ketter TA; SPD417 Study Group. A multicenter, randomized, double-blind, placebo-controlled trial of extended-release carbamazepine capsules as monotherapy for bipolar disorder patients with manic or mixed episodes. J Clin Psychiatry 2004, 65:478-484, doi: 10.4088/jcp.v65n0405

51. Weisler RH, Keck PE Jr, Swann AC, Cutler AJ, Ketter TA, Kalali AH et al. Extended-release carbamazepine capsules as monotherapy for acute mania in bipolar disorder: a multicenter, randomized, double-blind, placebo-controlled trial. J Clin Psychiatry 2005, 66:323-330, doi: 10.4088/ jcp.v66n0308

52. Fountoulakis KN, Gonda X, Vieta E, Rihmer Z. Class effect of pharmacotherapy in bipolar disorder: fact or misbelief? Ann Gen Psychiatry 2011, 10:8, doi: 10.1186/1744-859X-10-8

53. Goldsmith DR, Wagstaff AJ, Ibbotson T, Perry CM. Lamotrigine: a review of its use in bipolar disorder. Drugs 2003, 63:2029-2050, doi: 10.2165/00003495-200363190-00009

54. Young AH, McElroy SL, Bauer M, Philips N, Chang W, Olausson B et al. A double-blind, placebo-controlled study of quetiapine and lithium monotherapy in adults in the acute phase of bipolar depression (EMBOLDEN I). J Clin Psychiatry 2010, 71:150-162, doi: 10.4088/JCP.08m04995gre

55. Bond DJ, Lam RW, Yatham LN. Divalproex sodium versus placebo in the treatment of acute bipolar depression: a systematic review and meta-analysis. J Affect Disord 2010, 124:228-234, doi: 10.1016/j. jad.2009.11.008

56. Smith LA, Cornelius VR, Azorin JM, Perugi G, Vieta E, Young AH et al. Valproate for the treatment of acute bipolar depression: systematic review and meta-analysis. J Affect Disord 2010, 122:1-9, doi: 10.1016/j. jad.2009.10.033

57. Post RM, Uhde TW, Ballenger JC, Chatterji DC, Greene RF, Bunney WE Jr. Carbamazepine and its -10,11-epoxide metabolite in plasma and CSF. Relationship to antidepressant response. Arch Gen Psychiatry 1983, 40:673-676, doi: 10.1001/archpsyc.1983.04390010083010

58. Post RM, Uhde TW, Roy-Byrne PP, Joffe RT. Antidepressant effects of carbamazepine. Am J Psychiatry 1986, 143:29-34, doi: 10.1176/ajp. 143.1.29

59. Robertson B, Grunze H, Versavel M, Costa R, Almeida L, Soares-da-Silva P. Results of a double-blind, randomized, dose-titration, placebo controlled multicenter trial (SCO/BIA-2093-203 study) on safety and efficacy of eslicarbazepine acetate (BIA 2-093) for acute manic episodes associated with biopolar I disorder. Bipolar disord 2010, 12:46

60. Frye MA, Ketter TA, Kimbrell TA, Dunn RT, Speer AM, Osuch EA et al. A placebo-controlled study of lamotrigine and gabapentin monotherapy in refractory mood disorders. J Clin Psychopharmacol 2000, 20:607-614, doi: 10.1097/00004714-200012000-00004

61. Lombardo I, Sachs G, Kolluri S, Kremer C, Yang R. Two 6-week, randomized, double-blind, placebo-controlled studies of ziprasidone in outpatients with bipolar I depression: did baseline characteristics impact trial outcome? J Clin Psychopharmacol 2012, 32:470-478, doi: 10.1097/ JCP.0b013e31825ccde5

62. Calabrese JR, Keck PE Jr, Macfadden W, Minkwitz M, Ketter TA, Weisler $\mathrm{RH}$ et al. A randomized, double-blind, placebo-controlled trial of quetiapine in the treatment of bipolar I or II depression. Am J Psychiatry 2005 162:1351-1360, doi: 10.1176/appi.ajp.162.7.1351

63. Li H, Gu N, Zhang H, Wang G, Tan Q, Yang F et al. Efficacy and safety of quetiapine extended release monotherapy in bipolar depression: a multi-center, randomized, double-blind, placebo-controlled trial. Psychopharmacology (Berl) 2016, 233:1289-1297, doi: 10.1007/s00213016-4215-z

64. McElroy SL, Weisler RH, Chang W, Olausson B, Paulsson B, Brecher M et al. A double-blind, placebo-controlled study of quetiapine and paroxetine 
as monotherapy in adults with bipolar depression (EMBOLDEN II). J Clin Psychiatry 2010, 71:163-174, doi: 10.4088/JCP.08m04942gre

65. Suppes T, Datto C, Minkwitz M, Nordenhem A, Walker C, Darko D. Effectiveness of the extended release formulation of quetiapine as monotherapy for the treatment of acute bipolar depression. $J$ Affect Disord 2010, 121:106-115, doi: 10.1016/j.jad.2009.10.007

66. Thase ME, Macfadden W, Weisler RH, Chang W, Paulsson B, Khan A et al. Efficacy of quetiapine monotherapy in bipolar I and II depression: a double-blind, placebo-controlled study (the BOLDER II study). J Clin Psychopharmacol 2006, 26:600-609, doi: 10.1097/01. jcp.0000248603.76231.b7

67. Fountoulakis KN, Yatham LN, Grunze H, Vieta E, Young AH, Blier P et al. The CINP Guidelines on the Definition and Evidence-Based Interventions for Treatment-Resistant Bipolar Disorder. Int J Neuropsychopharmacol 2020, 23:230-256, doi: 10.1093/ijnp/pyz064

68. Henter ID, Park LT, Zarate CA Jr. Novel Glutamatergic Modulators for the Treatment of Mood Disorders: Current Status. CNS Drugs 2021, 35:527543, doi: 10.1007/s40263-021-00816-x

69. Lener MS, Kadriu B, Zarate CA Jr. Ketamine and Beyond: Investigations into the Potential of Glutamatergic Agents to Treat Depression. Drugs 2017, 77:381-401, doi: 10.1007/s40265-017-0702-8

70. Alberich S, Martínez-Cengotitabengoa M, López P, Zorrilla I, Núñez N, Vieta E et al. Efficacy and safety of ketamine in bipolar depression: A systematic review. Rev Psiquiatr Salud Ment 2017, 10:104-112, doi: 10.1016/j. rpsm.2016.05.005

71. Okada M, Kawano Y, Fukuyama K, Motomura E, Shiroyama T. Candidate Strategies for Development of a Rapid-Acting Antidepressant Class That Does Not Result in Neuropsychiatric Adverse Effects: Prevention of Ketamine-Induced Neuropsychiatric Adverse Reactions. Int J Mol Sci 2020, 21:7951, doi: 10.3390/ijms21217951

72. Jimerson DC, Post RM, Stoddard FJ, Gillin JC, Bunney WE Jr. Preliminary trial of the noradrenergic agonist clonidine in psychiatric patients. Biol Psychiatry 1980, 15:45-57, PMID: 7357058

73. Kastin AJ, Ehrensing RH, Schalch DS, Anderson MS. Improvement in mental depression with decreased thyrotropin response after administration of thyrotropin-releasing hormone. Lancet 1972, 2:740-742, doi: 10.1016/s0140-6736(72)92028-4

74. Osman OT, Rudorfer MV, Potter WZ. Idazoxan: a selective alpha 2-antagonist and effective sustained antidepressant in two bipolar depressed patients. Arch Gen Psychiatry 1989, 46:958-959, doi: 10.1001/archpsyc.1989.01810100100021

75. Kessler U, Vaaler AE, Schøyen H, Oedegaard KJ, Bergsholm P, Andreassen $\mathrm{OA}$ et al. The study protocol of the Norwegian randomized controlled trial of electroconvulsive therapy in treatment resistant depression in bipolar disorder. BMC Psychiatry 2010, 10:16, doi: 10.1186/1471-244X-10-16

76. Schoeyen HK, Kessler U, Andreassen OA, Auestad BH, Bergsholm P, Malt UF,et al. Treatment-resistant bipolar depression: a randomized controlled trial of electroconvulsive therapy versus algorithm-based pharmacological treatment. Am J Psychiatry 2015, 172:41-51, doi: 10.1176/ appi.ajp.2014.13111517

77. Dell'Osso B, Mundo E, D'Urso N, Pozzoli S, Buoli M, Ciabatti M et al. Augmentative repetitive navigated transcranial magnetic stimulation (rTMS) in drug-resistant bipolar depression. Bipolar Disord 2009, 11:76-81, doi: 10.1111/j.1399-5618.2008.00651.x

78. Nahas Z, Kozel FA, Li X, Anderson B, George MS. Left prefrontal transcranial magnetic stimulation (TMS) treatment of depression in bipolar affective disorder: a pilot study of acute safety and efficacy. Bipolar Disord 2003, 5:40-47, doi: 10.1034/j.1399-5618.2003.00011.x

79. Wu JC, Kelsoe JR, Schachat C, Bunney BG, DeModena A, Golshan S et al. Rapid and sustained antidepressant response with sleep deprivation and chronotherapy in bipolar disorder. Biol Psychiatry 2009, 66:298-301, doi: 10.1016/j.biopsych.2009.02.018

80. Dauphinais DR, Rosenthal JZ, Terman M, DiFebo HM, Tuggle C, Rosenthal NE. Controlled trial of safety and efficacy of bright light therapy vs. negative air ions in patients with bipolar depression. Psychiatry Res 2012, 196:57-61, doi: 10.1016/j.psychres.2012.01.015

81. Tohen M, Vieta E, Calabrese J, Ketter TA, Sachs G, Bowden C et al. Efficacy of olanzapine and olanzapine-fluoxetine combination in the treatment of bipolar I depression. Arch Gen Psychiatry 2003, 60:1079-1088, doi: 10.1001/archpsyc.60.11.1079

82. Sachs GS, Nierenberg AA, Calabrese JR, Marangell LB, Wisniewski SR Gyulai $L$ et al. Effectiveness of adjunctive antidepressant treatment for bipolar depression. N Engl J Med 2007 356:1711-1722, doi: 10.1056/ NEJMoa064135

83. Zhang ZJ, Kang WH, Tan QR, Li Q, Gao CG, Zhang FG et al. Adjunctive herbal medicine with carbamazepine for bipolar disorders: A double-blind, randomized, placebo-controlled study. J Psychiatr Res 2007, 41:360-369, doi: 10.1016/j.jpsychires.2005.06.002

84. van der Loos ML, Mulder $\mathrm{P}$, Hartong EG, Blom MB, Vergouwen AC, van Noorden MS et al. Efficacy and safety of two treatment algorithms in bipolar depression consisting of a combination of lithium, lamotrigine or placebo and paroxetine. Acta Psychiatr Scand 2010, 122:246-254, doi: 10.1111/j.1600-0447.2009.01537.x

85. van der Loos ML, Mulder $\mathrm{P}$, Hartong EG, Blom MB, Vergouwen AC, van Noorden MS et al. Long-term outcome of bipolar depressed patients receiving lamotrigine as add-on to lithium with the possibility of the addition of paroxetine in nonresponders: a randomized, placebo-controlled trial with a novel design. Bipolar Disord 2011, 13:111-117, doi: 10.1111/j.1399-5618.2011.00887.x

86. van der Loos ML, Mulder PG, Hartong EG, Blom MB, Vergouwen AC, de Keyzer $\mathrm{HJ}$ et al. Efficacy and safety of lamotrigine as add-on treatment to lithium in bipolar depression: a multicenter, double-blind, placebo-controlled trial. J Clin Psychiatry 2009, 70:223-231, doi: 10.4088/ jcp.08m04152

87. Bocchetta A, Bernardi F, Burrai C, Pedditzi M, Del Zompo M. A double-blind study of L-sulpiride versus amitriptyline in lithium-maintained bipolar depressives. Acta Psychiatr Scand 1993, 88:434-439, doi: 10.1111/ j.1600-0447.1993.tb03487.x

88. Juruena MF, Ottoni GL, Machado-Vieira R, Carneiro RM, Weingarthner N, Marquardt AR et al. Bipolar I and II disorder residual symptoms: oxcarbazepine and carbamazepine as add-on treatment to lithium in a double-blind, randomized trial. Prog Neuropsychopharmacol Biol Psychiatry 2009, 33:94-99, doi: 10.1016/j.pnpbp.2008.10.012

89. Diazgranados N, Ibrahim L, Brutsche NE, Newberg A, Kronstein P, Khalife $\mathrm{S}$ et al. A randomized add-on trial of an N-methyl-D-aspartate antagonist in treatment-resistant bipolar depression. Arch Gen Psychiatry 2010, 67:793-802, doi: 10.1001/archgenpsychiatry.2010.90

90. Loebel A, Cucchiaro J, Silva R, Kroger H, Sarma K, Xu J et al. Lurasidone as adjunctive therapy with lithium or valproate for the treatment of bipolar I depression: a randomized, double-blind, placebo-controlled study. Am J Psychiatry 2014, 171:169-177, doi: 10.1176/appi.ajp.2013.13070985

91. Xu AJ, Niciu MJ, Lundin NB, Luckenbaugh DA, lonescu DF, Richards EM et al. Lithium and Valproate Levels Do Not Correlate with Ketamine's Antidepressant Efficacy in Treatment-Resistant Bipolar Depression. Neural Plast 2015, 2015:858251, doi: 10.1155/2015/858251

92. Young LT, Joffe RT, Robb JC, MacQueen GM, Marriott M, Patelis-Siotis I. Double-blind comparison of addition of a second mood stabilizer versus an antidepressant to an initial mood stabilizer for treatment of patients with bipolar depression. Am J Psychiatry 2000, 157:124-126, doi: 10.1176/ajp.157.1.124

93. Zarate CA Jr, Brutsche NE, Ibrahim L, Franco-Chaves J, Diazgranados N, Cravchik A et al. Replication of ketamine's antidepressant efficacy in bipolar depression: a randomized controlled add-on trial. Biol Psychiatry 2012, 71:939-946, doi: 10.1016/j.biopsych.2011.12.010

94. Fountoulakis KN, Gonda X, Baghai TC, Baldwin DS, Bauer M, Blier P et al. Report of the WPA section of pharmacopsychiatry on the relationship of antiepileptic drugs with suicidality in epilepsy. Int J Psychiatry Clin Pract 2015, 19:158-167, doi: 10.3109/13651501.2014.1000930

95. Fountoulakis KN, Kontis D, Gonda X, Siamouli M, Yatham LN. Treatment of mixed bipolar states. Int J Neuropsychopharmacol 2012, 15:1015-1026, doi: 10.1017/S1461145711001817

96. Siamouli M, Samara M, Fountoulakis KN. Is antiepileptic-induced suicidality a data-based class effect or an exaggeration? A comment on the literature. Harv Rev Psychiatry 2014, 22:379-381, doi: 10.1097/ HRP.0000000000000039 
97. Altshuler LL, Post RM, Hellemann G, Leverich GS, Nolen WA, Frye MA et al. Impact of antidepressant continuation after acute positive or partial treatment response for bipolar depression: a blinded, randomized study. J Clin Psychiatry 2009, 70:450-457, doi: 10.4088/jcp.08m04191

98. Post RM, Altshuler LL, Frye MA, Suppes T, Rush AJ, Keck PE Jr et al. Rate of switch in bipolar patients prospectively treated with second-generation antidepressants as augmentation to mood stabilizers. Bipolar Disord 2001, 3:259-265, PMID: 11912569

99. Post RM, Altshuler LL, Leverich GS, Frye MA, Nolen WA, Kupka RW et al. Mood switch in bipolar depression: comparison of adjunctive venlafaxine, bupropion and sertraline. Br J Psychiatry 2006, 189:124-131, doi: 10.1192/bjp.bp.105.013045

100. Sachs GS, Ice KS, Chappell PB, Schwartz JH, Gurtovaya O, Vanderburg DG et al. Efficacy and safety of adjunctive oral ziprasidone for acute treatment of depression in patients with bipolar I disorder: a randomized, double-blind, placebo-controlled trial. J Clin Psychiatry 2011, 72:1413-1422, doi: 10.4088/JCP.09m05934

101. Sachs GS, Lafer B, Stoll AL, Banov M, Thibault AB, Tohen M et al. A double-blind trial of bupropion versus desipramine for bipolar depression. J Clin Psychiatry 1994, 55:391-393, PMID: 7929019

102. Saricicek A, Maloney K, Muralidharan A, Ruf B, Blumberg HP, Sanacora $G$ et al. Levetiracetam in the management of bipolar depression: a randomized, double-blind, placebo-controlled trial. J Clin Psychiatry 2011, 72:744-750, doi: 10.4088/JCP.09m05659gre

103. Schaffer A, Zuker P, Levitt A. Randomized, double-blind pilot trial comparing lamotrigine versus citalopram for the treatment of bipolar depression. J Affect Disord 2006, 96:95-99, doi: 10.1016/j.jad. 2006.05.02

104. Shelton RC, Stahl SM. Risperidone and paroxetine given singly and in combination for bipolar depression. J Clin Psychiatry 2004, 65:1715-1719, doi: 10.4088/jcp.v65n1218

105. McElroy SL, Martens BE, Mori N, Blom TJ, Casuto LS, Hawkins JM et al. Adjunctive lisdexamfetamine in bipolar depression: a preliminary randomized, placebo-controlled trial. Int Clin Psychopharmacol 2015, 30:6-13, doi: 10.1097/YIC.0000000000000051

106. Anand A, Gunn AD, Barkay G, Karne HS, Nurnberger Jl, Mathew SJ et al. Early antidepressant effect of memantine during augmentation of lamotrigine inadequate response in bipolar depression: a double-blind, randomized, placebo-controlled trial. Bipolar Disord 2012, 14:64-70, doi: 10.1111/j.1399-5618.2011.00971.x

107. Lee SY, Chen SL, Chang YH, Chen PS, Huang SY, Tzeng NS et al. The effects of add-on low-dose memantine on cytokine levels in bipolar II depression: a 12-week double-blind, randomized controlled trial. J Clin Psychopharmacol2014,34:337-343, doi: 10.1097/JCP.0000000000000109

108. Lee SY, Chen SL, Chang YH, Chen SH, Chu CH, Huang SY, Tzeng NS et al. Genotype variant associated with add-on memantine in bipolar II disorder. Int J Neuropsychopharmacol 2014, 17:189-197, doi: 10.1017/ S1461145713000825

109. Abdallah CG, Fasula M, Kelmendi B, Sanacora G, Ostroff R. Rapid antidepressant effect of ketamine in the electroconvulsive therapy setting. J ECT 2012, 28:157-161, doi: 10.1097/YCT.0b013e31824f8296

110. Brown ES, Davila D, Nakamura A, Carmody TJ, Rush AJ, Lo A et al. A randomized, double-blind, placebo-controlled trial of quetiapine in patients with bipolar disorder, mixed or depressed phase, and alcohol dependence. Alcohol Clin Exp Res 2014, 38:2113-2118, doi: 10.1111/ acer.12445

111. Bauer M, Berman S, Stamm T, Plotkin M, Adli M, Pilhatsch M et al. Levothyroxine effects on depressive symptoms and limbic glucose metabolism in bipolar disorder: a randomized, placebo-controlled positron emission tomography study. Mol Psychiatry 2016, 21:229-236, doi: 10.1038/mp.2014.186

112. Chiu CC, Huang SY, Chen CC, Su KP. Omega-3 fatty acids are more beneficial in the depressive phase than in the manic phase in patients with bipolar I disorder. J Clin Psychiatry 2005, 66:1613-1614, doi: 10.4088/ jcp.v66n1219b

113. Frangou S, Lewis M, Wollard J, Simmons A. Preliminary in vivo evidence of increased $\mathrm{N}$-acetyl-aspartate following eicosapentanoic acid treatment in patients with bipolar disorder. J Psychopharmacol 2007, 21:435-439, doi: 10.1177/0269881106067787

114. Keck PE Jr, Mintz J, McElroy SL, Freeman MP, Suppes T, Frye MA. Double-blind, randomized, placebo-controlled trials of ethyl-eicosapentanoate in the treatment of bipolar depression and rapid cycling bipolar disorder. Biol Psychiatry 2006, 60:1020-1022, doi: 10.1016/j.biopsych.2006.03.056

115. Murphy BL, Stoll AL, Harris PQ, Ravichandran C, Babb SM, Carlezon WA Jr et al. Omega-3 fatty acid treatment, with or without cytidine, fails to show therapeutic properties in bipolar disorder: a double-blind, randomized add-on clinical trial. J Clin Psychopharmacol 2012, 32:699-703, doi: 10.1097/JCP.0b013e318266854c

116. Sarris J, Mischoulon D, Schweitzer I. Omega-3 for bipolar disorder: meta-analyses of use in mania and bipolar depression. J Clin Psychiatry 2012, 73:81-86, doi: 10.4088/JCP.10r06710

117. Stoll AL, Severus WE, Freeman MP, Rueter S, Zboyan HA, Diamond E et al. Omega 3 fatty acids in bipolar disorder: a preliminary double-blind, placebo-controlled trial. Arch Gen Psychiatry 1999, 56:407-412, doi: 10.1001/archpsyc.56.5.407

118. Sylvia LG, Peters AT, Deckersbach T, Nierenberg AA. Nutrient-based therapies for bipolar disorder: a systematic review. Psychother Psychosom 2013, 82:10-19, doi: 10.1159/000341309

119. Amsterdam JD, Garcia-Espana F. Venlafaxine monotherapy in women with bipolar II and unipolar major depression. J Affect Disord 2000, 59:225-229, doi: 10.1016/s0165-0327(99)00149-4

120. Baumhackl U, Bizière K, Fischbach R, Geretsegger C, Hebenstreit G, Radmayr $\mathrm{E}$ et al. Efficacy and tolerability of moclobemide compared with imipramine in depressive disorder (DSM-III): an Austrian double-blind, multicentre study. Br J Psychiatry Suppl 1989, 78-83, PMID: 2695130

121. Cohn JB, Collins G, Ashbrook E, Wernicke JF. A comparison of fluoxetine imipramine and placebo in patients with bipolar depressive disorder. Int Clin Psychopharmacol 1989, 4:313-322, doi: 10.1097/ 00004850198910000-00006

122. Grossman F, Potter WZ, Brown EA, Maislin G. A double-blind study comparing idazoxan and bupropion in bipolar depressed patients. J Affect Disord 1999, 56:237-243, doi: 10.1016/s0165-0327(99)00041-5

123. Himmelhoch JM, Thase ME, Mallinger AG, Houck P. Tranylcypromine versus imipramine in anergic bipolar depression. Am J Psychiatry 1991, 148:910-916, doi: 10.1176/ajp.148.7.910

124. Silverstone T. Moclobemide vs. imipramine in bipolar depression: a multicentre double-blind clinical trial. Acta Psychiatr Scand 2001, 104:104-109, doi: 10.1034/j.1600-0447.2001.00240.x

125. Vieta E, Martinez-Arán A, Goikolea JM, Torrent C, Colom F, Benabarre A et al. A randomized trial comparing paroxetine and venlafaxine in the treatment of bipolar depressed patients taking mood stabilizers. J Clin Psychiatry 2002, 63:508-512, doi: 10.4088/jcp.v63n0607

126. Thase ME, Mallinger AG, McKnight D, Himmelhoch JM. Treatment of imipramine-resistant recurrent depression, IV: A double-blind crossover study of tranylcypromine for anergic bipolar depression. Am J Psychiatry 1992, 149:195-198, doi: 10.1176/ajp.149.2.195

127. McClure D, Greenman SC, Koppolu SS, Varvara M, Yaseen ZS, Galynker II. A Pilot Study of Safety and Efficacy of Cranial Electrotherapy Stimulation in Treatment of Bipolar II Depression. J Nerv Ment Dis 2015, 203:827-835, doi: $10.1097 /$ NMD.0000000000000378

128. Lorenzo-Luaces L, Amsterdam JD, Soeller I, DeRubeis RJ. Rapid versus non-rapid cycling bipolar II depression: response to venlafaxine and lithium and hypomanic risk. Acta Psychiatr Scand 2016, 133:459-469, doi: 10.1111/acps.12557

129. Taylor D, Barnes TRE, Young AH. The Maudsley prescribing guidelines in psychiatry. 13th Ed, Wiley-Blackwell 2019 


\title{
Clinical characteristics and treatment of treatment-resistant bipolar depression
}

\author{
Grigorios N. Karakatsoulis, ${ }^{1}$ Eva-Maria Tsapakis, ${ }^{2,3}$ Konstantinos N. Fountoulakis' \\ 13rd Department of Psychiatry, Aristotle University of Thessaloniki, University General Hospital AHEPA, Thessaloniki, \\ ${ }^{2} 1$ st Department of Psychiatry, Aristotle University of Thessaloniki, Papageorgiou General Hospital, Thessaloniki, \\ 3"Agios Charalambos" Mental Health Clinic, Heraklion, Crete, Greece
}

\begin{abstract}
Depression represents the predominant mood pole in bipolar disorder. Bipolar depression typically has a poor response to antidepressant medication, and also involves the risk of polarity shifts, induction of mixed states, and / or rapid cycle induction. The diagnosis of bipolar depression can be delayed by 8 to 10 years. The reason for this delay is mainly the fact that both manic and hypomanic episodes appear lately in the course of the disorder. It is therefore necessary to diagnose this clinical entity as early as possible versus monopolar depression in order to treat it more effectively. This differential diagnosis is based on certain clinical features of bipolar depression, which are often difficult to be distinguished from those of monopolar depression and therefore it is necessary to know specific criteria that differentiate them to some extent qualitatively and / or quantitatively. Such characteristics are daily mood swings, multiple physical complaints, psychomotor retardation, psychotic elements (delusions and perceptual disorders mood congruent or noncongruent), the disturbance of certain bodily functions, including circadian rhythms, sexual desire, appetite, and disorders of sleep architecture. The treatment of bipolar depression is based on the options known from monopolar depression (such as the use of antidepressants, antipsychotics, and certain antiepileptic agents) and their combinations, while in recent years it has been enriched with new pharmaceutical agents and non-pharmacological approaches. New glutaminergic regulators dominate the new pharmacological agents' research, and among them the antidepressant effect of ketamine and esketamine at sub-anesthetic doses is being extensively investigated during recent years. Non-pharmacological approaches include methods such as electroconvulsive therapy, repetitive transcranial magnetic stimulation (rTMS), sleep deprivation, and phototherapy.
\end{abstract}

KEYWORDS: Bipolar depression, clinical presentation, differential diagnosis, pharmaceutical therapy, non- pharmaceutical therapies.

Corresponding author: Grigorios N. Karakatsoulis, Department of Psychiatry, Aristotle University of Thessaloniki, University General Hospital AHEPA, 1 Stilponos Kyriakidi street, GR-543 51 Thessaloniki, Greece•e-mail: gregkarakatsoulis@gmail.com 